\title{
The Penetration of Internet of Things in Robotics: Towards a Web of Robotic Things
}

\author{
Andreas Kamilaris ${ }^{\mathrm{a}, \mathrm{b}}$, Nicolò Botteghic \\ ${ }^{a}$ Research Centre on Interactive Media, Smart Systems and Emerging Technologies (RISE), Nicosia, Cyprus \\ ${ }^{b}$ Dept. of Computer Science, University of Twente, Enschede, The Netherlands \\ ${ }^{c}$ Dept. of Electrical Engineering, University of Twente, Enschede, The Netherlands
}

\begin{abstract}
As the Internet of Things (IoT) penetrates different domains and application areas, it has recently entered also the world of robotics. Robotics constitutes a modern and fast-evolving technology, increasingly being used in industrial, commercial and domestic settings. IoT, together with the Web of Things (WoT) could provide many benefits to robotic systems. Some of the benefits of IoT in robotics have been discussed in related work. This paper moves one step further, studying the actual current use of IoT in robotics, through various real-world examples encountered through a bibliographic research. The paper also examines the potential of WoT, together with robotic systems, investigating which concepts, characteristics, architectures, hardware, software and communication methods of IoT are used in existing robotic systems, which sensors and actions are incorporated in IoT-based robots, as well as in which application areas. Finally, the current application of WoT in robotics is examined and discussed.
\end{abstract}

Keywords: Internet of Things; Web of Things; Robotics; Robots; Sensors. 


\section{Introduction}

The Internet of things (IoT) [128] is the extension of Internet connectivity into physical devices and everyday objects. As the IoT penetrates different domains, application areas and scientific disciplines [76], it is worth the effort to examine its impact, interaction and application in the research area of robotics.

Robotics constitutes a modern and fast-evolving technology [102], which is increasingly being used in industrial, commercial and domestic settings, as well as for rescue operations where there are safety risks for humans [121]. Robotics can be defined as the branch of engineering that involves the conception, design, manufacture and operation of robots [32]. Robot comes from the Czech word robota which means forced work or labour. The word robot today means any man-made machine that can perform work or other actions normally performed by humans, either automatically or by remote control. Robots are employed because it is often cheaper to use them over humans, easier for robots to do some job and sometimes the only possible way to accomplish some tasks. Most robots are composed of a controller (i.e. the brain of the robot), mechanics (i.e. motors, pistons, grippers, wheels and gears that make the robot move, grab, turn, and lift) and sensors, i.e. to help the robot perceive its surroundings. An example of a robot with a human-like shape is provided in Figure 1. Although robots have been mostly used in industrial applications till date, recent technological progress in the emerging domains of cognition, manipulation and interactions is moving the robotics industry toward service robots and human-centric design [131].

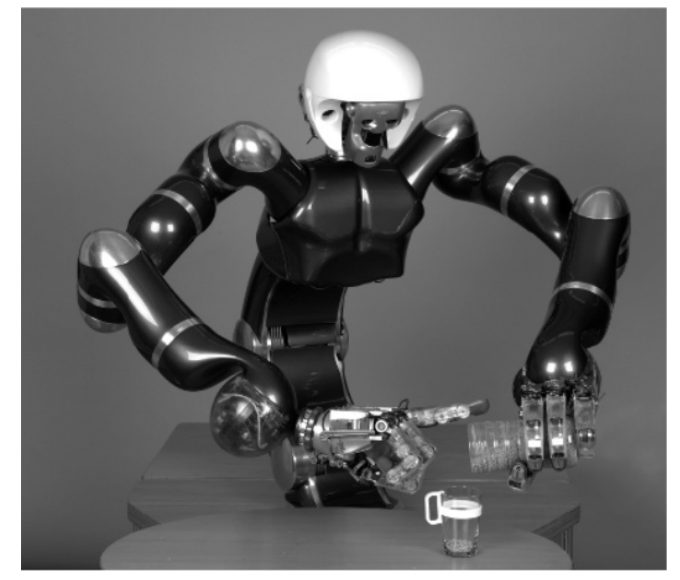

Figure 1: An example robot pouring some liquid from one cup to another (Source: [102]).

The interconnection and relationship between the IoT and robotics has been defined as Internet of Robotic Things (IoRT) [91, 120, 105]. IoRT is about a global infrastructure enabling advanced robotic services by interconnecting robotic things based on, existing and evolving, interoperable information and communication technologies such as cloud computing, cloud storage and other existing Internet technologies. IoT allows robots to communicate by means of the IP protocol, especially its IPv6 version, which is designed for billions of Internet-connected objects [48]. Internet connection permits updating information (and possibly the robot's firmware) in real-time [118], storing/processing data on the cloud and taking advantage of Internet protocols for security, authentication, data integrity, message routing etc. [106, 110].

We argue that IoRT should also embrace the Web of Things (WoT) [129, 39], which is about approaches, software architectural styles and programming patterns that allow real-world objects to be part of the World Wide Web. WoT could offer additional benefits to the IoT-enabling of robotic-based systems, especially in terms of higher interoperability among the robot's sensory components, but also robot-to-robot (R2R) and robot-to-human (R2H) communication at the application layer. Additionally, WoT could facilitate the combination of Web services and robotic services, towards physical mashups [38], which could evolve to robotic mashups, realizing the notion of a Web of Robotic Things (WoRT). These benefits are discussed in Section 5, in more detail, together with a small historical journey on the first robots that had presence and allowed remote control via the Web, back in 1995 .

The main motivation for this paper comes from the observation on how other research areas and/or application domains have been influenced in the past by the introduction of IoT and WoT. These areas include home automation [109, 56], smart buildings [17], smart grid [21], remote healthcare [132], agriculture ,[58] as well as a limited number 
of smart city applications [59, 55]. Examples of this influence by IoT/WoT involve reduced risks of vendor lockin, adopting machinery (i.e. for agriculture) and sensing/automation systems from different companies, as these could become easily interoperable in the overall smart systems, easier data exchange among different, heterogeneous components, increased automation with less effort by means of Internet and Web standards etc. Use of open standards brings seamless connectivity and advanced interoperability, while the publishing of data produced by IoT sensors as open data on the Web promotes knowledge sharing and is important for the advancement of research in these fields.

Additional motivation for preparing this paper stems from the fact that related work in this field [120, 105, 91, 94, 37] has discussed only generally some of the opportunities of IoT in robotics. There is a gap in literature in relation to the actual degree of penetration of IoT in robotic systems and services, as well as how IoT has been used in robot systems till date. In addition, the current application and future potential of WoT in robotics has not been discussed in any relevant paper, except from [37], where the aspect of semantic consensus has been generally discussed, suggesting that this issue could be approached via Semantic Web technologies. In particular, the work in [105] focuses on the lower and higher level abilities of IoT-enabled robots, while Vermesan et al. [120] discuss generally some technologies of IoT that can support robotic systems. Ray [91] lists some examples of existing robots envisaged for an IoRT architecture, but the linking between the examples mentioned in the paper with the IoT is unclear and not convincing. The differences between this survey and relevant ones are summarized in Table 1 .

Table 1: Diffferences between this survey and related ones.

\begin{tabular}{|c|c|c|c|c|c|c|}
\hline Characteristic/Paper & [91] & [120] & [105] & [94] & [37] & $\begin{array}{c}\text { Our } \\
\text { paper }\end{array}$ \\
\hline $\begin{array}{l}\text { Review of papers demon- } \\
\text { strating robots }\end{array}$ & $\begin{array}{l}\text { IoRT- } \\
\text { based } \\
\text { ones }\end{array}$ & & Only general & $\begin{array}{l}\text { Only } \\
\text { general }\end{array}$ & Only general & $\begin{array}{l}\text { IoRT- } \\
\text { based } \\
\text { ones }\end{array}$ \\
\hline $\begin{array}{l}\text { Description of IoRT Tech- } \\
\text { nologies }\end{array}$ & $\mathrm{X}$ & $\begin{array}{c}\text { Focus on } \\
\text { software } \\
\text { platforms and } \\
\text { interoperabil- } \\
\text { ity }\end{array}$ & & $\begin{array}{l}\text { Only } \\
\text { cloud } \\
\text { robotics }\end{array}$ & $\begin{array}{l}\text { IoT security, } \\
\text { hardware } \\
\text { platforms }\end{array}$ & $\mathrm{X}$ \\
\hline $\begin{array}{l}\text { Linking between robots, } \\
\text { sensors, actions and appli- } \\
\text { cations }\end{array}$ & & & $\begin{array}{c}\text { Crossover of } \\
\text { IoRT into } \\
\text { nine robotic } \\
\text { system } \\
\text { abilities }\end{array}$ & $\begin{array}{l}\text { Link with } \\
\text { applica- } \\
\text { tions }\end{array}$ & Only general & $\mathrm{X}$ \\
\hline $\begin{array}{l}\text { Linking of robots with IoRT } \\
\text { technologies }\end{array}$ & & & & & $\begin{array}{l}\text { Link between } \\
\text { IoRT technologies } \\
\text { and EU projects }\end{array}$ & $\mathrm{X}$ \\
\hline Research challenges & $\mathrm{X}$ & & & & Only general & $\mathrm{X}$ \\
\hline $\begin{array}{l}\text { Presenting the whole picture } \\
\text { around IoRT }\end{array}$ & $\mathrm{X}$ & $\mathrm{X}$ & & & & $\mathrm{X}$ \\
\hline $\begin{array}{l}\text { Linking of robots with } \\
\text { WoRT technologies }\end{array}$ & & & & & $\begin{array}{c}\text { Semantic } \\
\text { consensus towards } \\
\text { Semantic WoT }\end{array}$ & $\mathrm{X}$ \\
\hline
\end{tabular}

Therefore, the contribution of this paper is to study the current use of IoT in robotics, through various real-world examples encountered through a bibliography-based research and to examine the possibilities of the WoT together with robotic systems. To the authors' knowledge, it is the most complete survey paper to date, aiming to present the actual research taking place in the intersection of these research domains.

The rest of this paper is organized as follows: Section 2 describes the methodology adopted and Section 3 introduces the concept of robotics, clarifying its relationship with IoT. Section 4 presents the existing penetration of IoT in 
robotics, while Section 5 studies the connection between WoT and robotic systems. Finally, Section 6 discusses the overall findings and Section 7 concludes the paper.

\section{Methodology}

This paper aims to fill the aforementioned gaps in literature, by addressing the following research questions:

1. Which concepts, characteristics, architectures, platforms, software, hardware and communication standards of IoT have been used by existing robotic systems and services?

2. Which sensors and actuators have been incorporated in IoT-based robots?

3. In which application areas has IoRT been applied?

4. Which technologies of IoT have been used in robotics till today?

5. Has WoT been used in robotics? If yes, by means of which technologies?

6. Which is the overall potential of WoT in combination with robotics, towards a WoRT?

As the IoT has been defined in different ways in literature, it must be clarified that IoT -in the context of this paperis perceived as a system that involves real-world things, which can communicate/interact over the Internet and they can be remotely monitored and controlled via Internet protocols. These devices involve robots and robotic systems in the context of this work.

Search for related work was performed through the Web scientific indexing services Web of Science and Google Scholar. The following query was used:

Robotics AND ["Internet of Things" OR "Web of Things"]

Thirty seven (37) papers were found via this approach. To increase the range of our bibliography, a search of the related work as appeared in these 37 papers was also performed. This effort allowed to increase the number of papers discovered to 61 . From these 61 papers, 12 papers $(20 \%)$ were discarded, as they did not involve a real-world implementation of a robotic system or because they have not used IoT or WoT somehow in their implementations. Forty nine (49) papers were finally selected in order to be analyzed in more detail. Each of these papers was studied in detail, aiming to address the aforementioned six research questions. The results of our research are presented in the next sections.

We note that this paper focuses on the connection between robotics and the concepts/principles of IoT and WoT, aiming to close the existing gap in literature, as described in the introductory section. This paper does not intend to compare specific hardware and software platforms used for sensors/robots development and/or development of IoT-based systems. For such comparisons, the reader should consider relevant studies [120, 75, 54, 19, 33, 93, 131], which cover quite well the spectrum of embedded hardware/software development, plus low-power communications. The research areas targeted by this paper are displayed as a Venn diagram in Figure 2 WoT is considered a subset of IoT and they both interact with robotics via the emerging research areas of IoRT and WoRT. Additional circles in the figure could be sensor technologies and communication protocols, as they are extensively used both by IoT and robotics.

We note that IoRT is mostly about technical aspects of robotic systems, as well as technologies for communication and message exchange or services and data understanding. It is not about artificial intelligence, robot perception and empathic behaviour, where robotics touches upon other research disciplines.

\section{Robotics}

Robots are becoming a fundamental part of our society and they will become even more important in future. The past decades were characterized by the massive automation in the industry, as for example in the case of automatic machines and industrial manipulators. In this context, the robots work in a perfectly known and modelled environment and safety layers are built around them to prevent harming people and other machinery. Furthermore, the actions of these robots are completely programmed in order to avoid any unpredictable behaviours. The robots work fast and accurately in order to improve the efficiency of industrial processes.

However, to further progress in the integration with everyday life, robots, usually referred as service robots in this context, need to be able to perceive and understand unknown and complex environments and to be capable of planning 


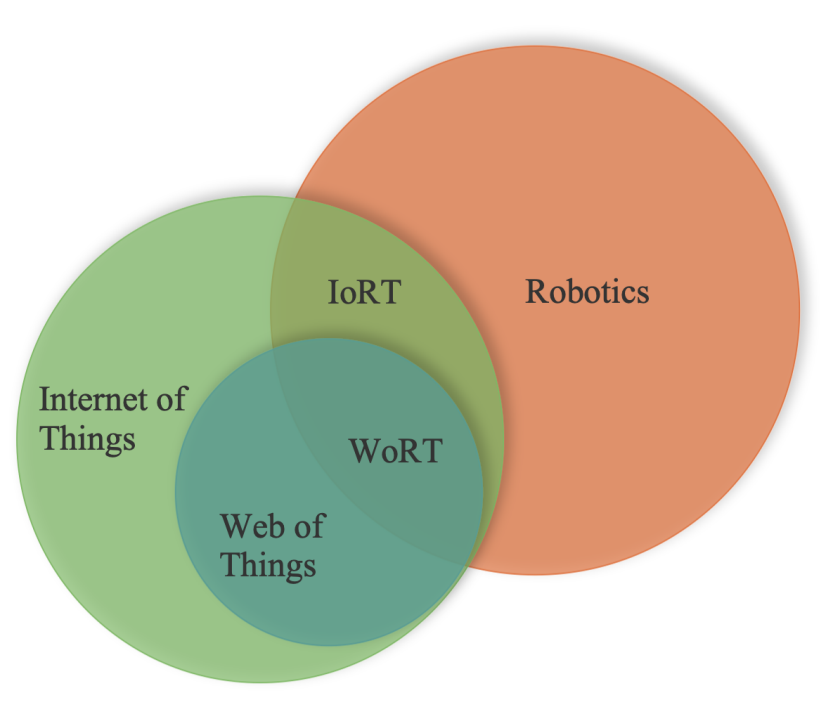

Figure 2: A Venn diagram showing research areas involved.

and acting in unforeseen situations. In robotics, these concepts are usually gathered together under the word cognition. Cognition is the key aspect in order to deal with the variety of environmental aspect, parameters and tasks in which the service robots operate, i.e. houses, warehouses and offices. The second major challenge is the manipulation of a high variety of objects, deformable or not, in the operational environment. The final hurdle for robots is the interaction. Robots and humans are expected to end up in the same environments, working in parallel and collaborating together, without any safety layer to keep them apart. Thus, the robots' behaviour must be predictable and safe for humans, for the surroundings and eventually for other co-operating robots. The concept of interaction, however, cannot be limited only to physical and safe interaction between humans and robots, but it must be extended to more abstract ways of interacting. Interpreting verbal and non-verbal communication such as as facial expression, body movements and gestures, as well as understanding of social interactions are clearly necessary steps to be taken in the near future.

\subsection{Internet of Things and Robotics}

Since robots are entering the everyday life of humans, supporting their tasks and interacting with them, the sensory equipment, hardware platforms, software and communication patterns of the robot machines become more complicated and demanding. As mentioned before, robots need to interact with their environment, i.e. other robots, devices and machines (R2R) and also with humans (R2H). To achieve this in a highly interoperable way towards true machine-to-machine (M2M) interaction involving context understanding, considering the wide variety of protocols and hardware/software/communication architectures and solutions available, the IoT and WoT come into play. The particular benefits from a WoT integration are listed in Section 3.2 below. The main difference between IoT and WoT is that IoT operates in the lower layers of the ISO stack, while WoT mainly at the application layer. Some overlap between IoT and WoT may exist at the presentation and session layers of the ISO stack. This difference is illustrated in Figure 3. The right part of the figure lists many of the technologies and acronyms mentioned through this survey, under the relevant part of the ISO stack where they belong, as well as whether they constitute mainly IoT or WoT technologies.

IoT could improve robotics with higher productivity (i.e. by re-using well-accepted and understood software and protocols), lower costs, better customer experiences due to the easier integration with existing components of the nearby environment and with cloud computing, high-quality data in terms of semantics awareness, context understanding and many other possibilities listed in related work [128, 129, 76].

Moreover, robotics can benefit from the plethora of research and development in IoT, in terms of resourceconstraint hardware and software, low-power communication algorithms and protocols, as well as optimal solutions for wireless sensor networks (WSN), such as networking, mobility, data propagation, topology building and maintenance etc. [92]. Finally, the unique naming and addressing capabilities provided by the IPv6 protocol, allow robots 

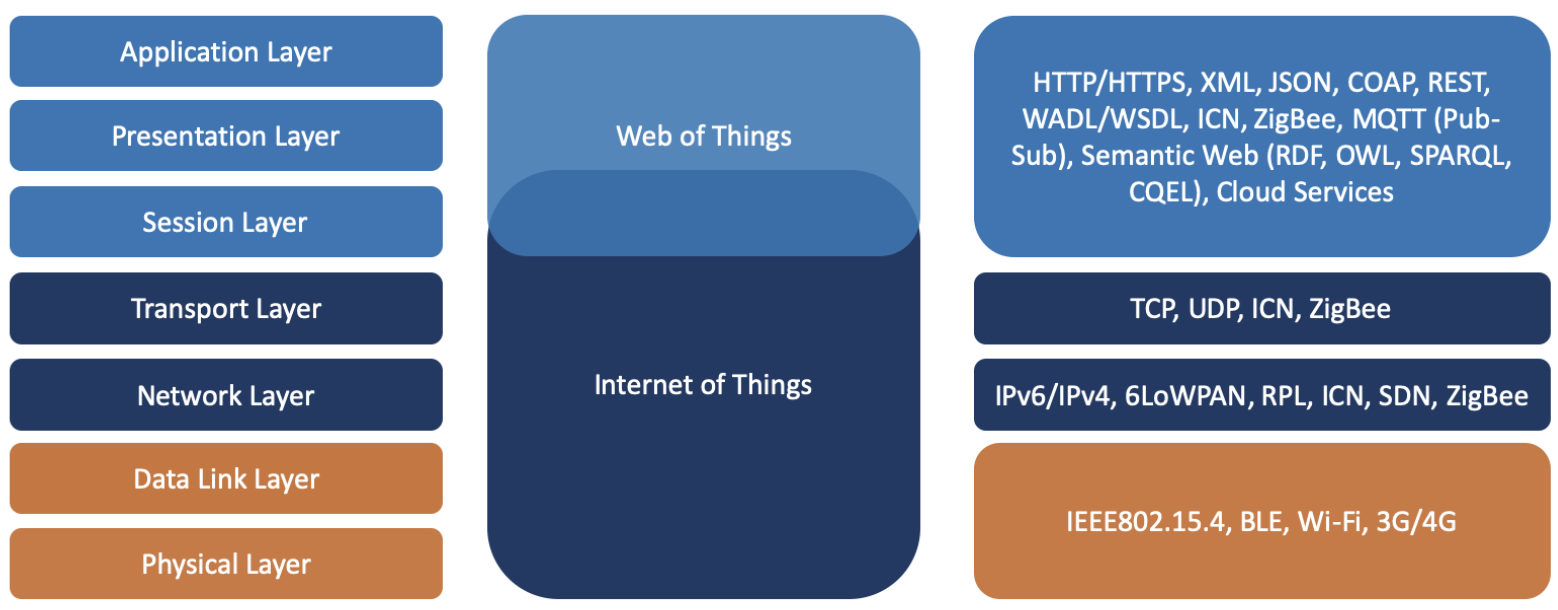

Figure 3: Layers of the ISO stack where IoT and WoT are located.

and robotic system to become uniquely addressable citizens of the Internet, exploiting the TCP/IP protocols for device discovery, message exchange, security etc.

\subsection{Web of Things and Robotics}

One of the first robots having a basic presence on the Web was MERCURY [35], which started its operation in 1994. It was one of the first tele-operated manipulators on the Web. It enabled Web users to excavate artefacts buried in a sand-filled terrarium. The TELE-GARDEN robot in 1995 [36], successor of MERCURY, allowed people to control the planting of flowers via a Web interface, being able to coordinate requests by multiple users. XAVIER followed in 2002 [103]. Although it had only a basic Web interface allowing only basic interaction possibilities, it became quite successful due to the possibilities of remote control of a robot via any Web browser around the world.

As mentioned in the introduction, WoT could offer more benefits to robotic systems than IoT alone [129, 39], especially in terms of interoperability at the application layer among robotic components and services, but also between robots (R2R) and humans (R2H), as well as among other machines (M2M). Some of these benefits are listed below, in more detail, in relation to robotic systems:

- Data from the sensors of the robots may be easily exported into Web applications in popular, well-understood standard formats, for easier reuse. Representation formats may be negotiated in real-time, depending on the formats supported by the machines involved.

- Exposing the services provided by the robots (and their individual sensing and actuating components) as interoperable application programming interfaces (APIs), would provide the primitives to users with little programming experience to perform advanced tasks. Users may select any programming language that supports the HTTP protocol, such as Python, Java, Ruby, C, PHP, JavaScript etc.

- Exposing robotic services as APIs would facilitate application-layer interoperability between robots (R2R) and humans (R2H). When these APIs become standardized, enhanced with semantic technologies, interaction between robots and humans can become automatic and in real-time, using the Web as the common platform and language for communication.

- Combining robotic services with Web services and resources would allow the creation of Web-based robotic mashups, where the robots exploit seamlessly knowledge, information and context already available at the Web, towards more informed choices and aware behaviour. 
- Uniform access to heterogeneous embedded devices installed on the robot, where the robot itself becomes a homogeneous environment (i.e. at the application layer), where any sensor/actuation can be individually accessed in a standardised way, facilitating coordination, action-taking and control. This homogeneous access would allow easier connection between robotic systems and cloud computing, for more advanced real-time computing processing and for permanent storage of information such as sensory measurements.

- Harnessing well-defined protocols used for years on the Web for device and service discovery, service and data description, semantics understanding, security and privacy, orchestration and routing.

- Particularly related to semantics of services and information, the Semantic Web (see Section 5.1 below) involves numerous technologies and implementations for uniformly describing devices, services and data, allowing common understanding and advanced reasoning between different entities.

We note that APIs in the WoT research area are expected to be resource-oriented and to follow the REpresentational State Transfer (REST) [30]. REST is an architectural style for developing Web Services and it has been adopted by WoT due to its simplicity and the fact that it builds upon existing systems and features of the HTTP protocol. A RESTful API follows the principles of REST for providing Web Services to the users, based on the HTTP request/response protocol. Thus, basic HTTP-based interfaces that do not follow REST can not be considered part of the WoT. However, they can still be considered part of the IoT, as long as they employ TCP/IP.

\section{Analysis of the Application of Internet of Things in Robotics}

This section addresses the first three research questions as defined in Section 2 First, Section 4.1 lists the existing applications of IoT in robotics, presenting how sensors and robot actions have been used in different application domains. Then, Section 4.2 shows some of the hardware elements and sensory equipment used in the work under study. Afterwards, Section 4.3 presents the software platforms and communication protocols used during programming and control of IoT-based robotic systems. Finally, Section 4.4 summarizes in which ways the IoT-enabled robots of related work have made actual use of Internet technologies.

\subsection{Applications}

Table 2 lists the different application domains and their specific application areas, where IoT-based robots and robotic services have been used. Most popular categories are entertainment (8 papers), health (7 papers), education (6 papers), surveillance (6 papers) and culture (5 papers). Some categories are overlapping, such as health with domestic support, surveillance with military, as well as emergency/disaster response with rescue operations. Autonomous cars are used as moving robots in the area of transportation, while a warfare robot car has been developed for military purposes in [47]. Unmanned aerial vehicles (UAV) (i.e. drones) are considered as flying robots in surveillance, emergency/disaster response and rescue operations.

Example robots as they appear in different application areas of the work under study are shown in Figure 4

Figure 5 maps together the sensing/actuation capabilities of the IoT robots, together with their actions, in relationship to the different application areas as encountered in this study by analyzing the related bibliography. The most popular sensors, actions and application areas are highlighted in blue color. As the figure shows, the most commonly used sensors are the ones that measure proximity (for the actions of touching, picking and placing, reminding), vision (for the actions of picking and placing, moving, observing, flying, spraying and pruning, reminding), voice (for the actions of speaking, listening and voice recognition) and motion (for the actions of picking and placing, speaking, observing, climbing, swimming and displaying text).

The most popular actions involve moving, observing, flying and navigating. Some actions are appropriate only for specific application areas, such as spray and prune for agriculture, reminders for health and domestic support, pick-and-place for customer support and industrial applications etc.

In relation to the possible actions performed by the robot, based on its sensory equipment, the application areas where most actions have been recorded are agriculture ( 7 actions), domestic support (6 actions), surveillance (6 actions), military (6 actions), rescue operations (5 actions) and entertainment (5 actions). 

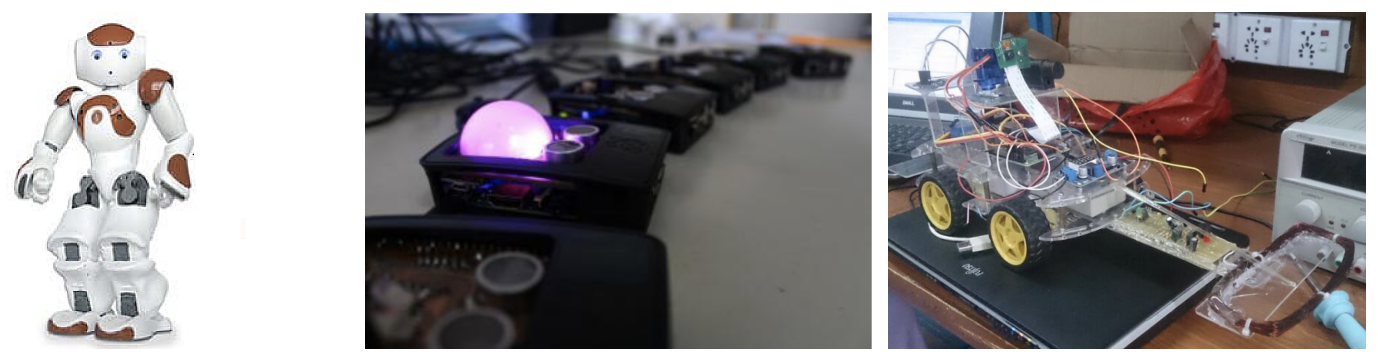

(a) Robot used for diabetes management(b) Robot for measuring people's re- (c) Robot car model used for warfare for children (Source: [7]).

flexes (Source: [26]).

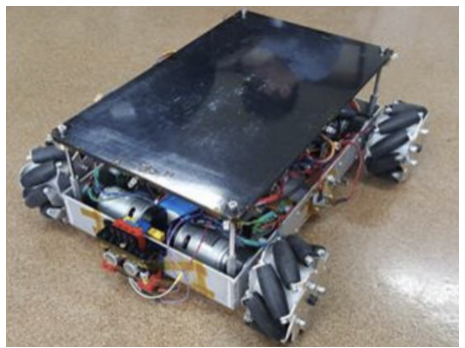

(d) Robot used for material handling at (e) Robot presenting pet behavior the industry (Source: [123]).

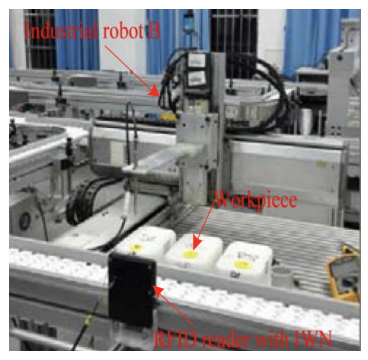

(Source: [61]).
(Source: [47]).

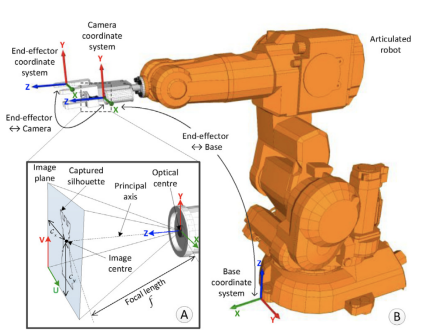

(f) Robot used in agriculture for crops' monitoring(Source: [126]).

Figure 4: Example robots as presented in related work under study.

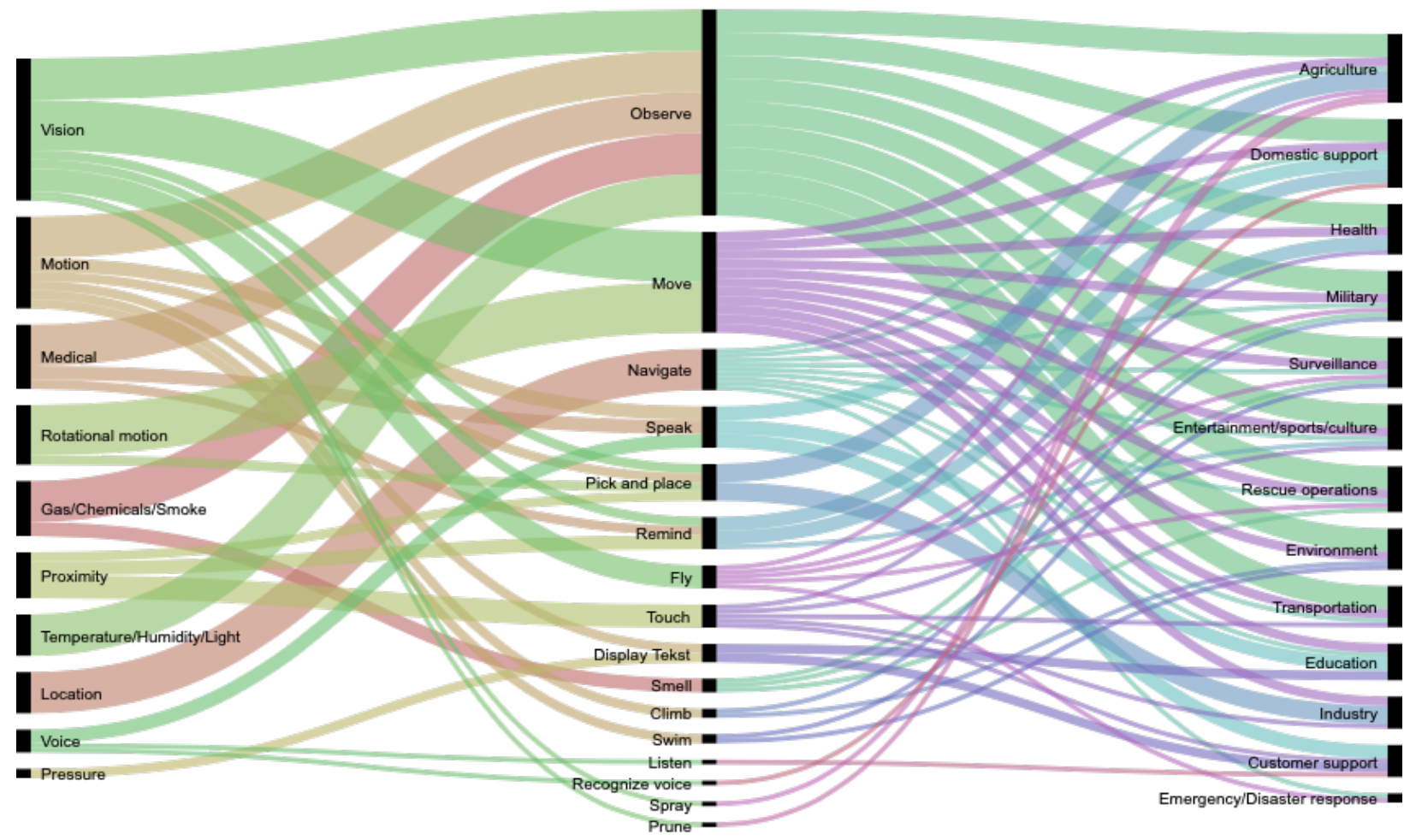

Figure 5: The connection between IoT robot sensors, actions and IoT application areas, as they appear in related work under study. 


\begin{tabular}{|c|c|}
\hline Application Area & Application \\
\hline Industry & Manufacturing [14, 123], material handling [124], 3D assembly operations [126] \\
\hline Customer support & Office operations' support [77, 103] \\
\hline Transportation & Autonomous cars [34], car parking system [49] \\
\hline Environment & $\begin{array}{l}\text { Water quality monitoring [125], smoke detection [11], air quality [119], space explo- } \\
\text { ration [10] }\end{array}$ \\
\hline Health & $\begin{array}{l}\text { Diabetes management [7, 6, 5], measuring reflexes [26], tele-surgery [18], tele- } \\
\text { echography [71], remote treatment [111] }\end{array}$ \\
\hline Education & $\begin{array}{l}\text { Teaching computing, programming and robotics in schools and universities [16, } 41 \text {, } \\
\text { 118], collaborative learning [88], educate people in public places [15] }\end{array}$ \\
\hline Domestic support & $\begin{array}{l}\text { Medical and health care [44], support of people with dementia [104], support of el- } \\
\text { derly and disabled residents [50], independent living [23] }\end{array}$ \\
\hline Entertainment & $\begin{array}{l}\text { Pet robot [61, 68], remote painting [107], robot that sings and dances [66], entertain } \\
\text { people in public places [15], allow low-cost public access to a tele-operated robot } \\
\text { [35], interact with a remote garden filled with living plants [36], moving in a wooden } \\
\text { labyrinth trying to get out of the maze [96] }\end{array}$ \\
\hline Sports & $\begin{array}{l}\text { Ball detection and catching [112], measuring reflexes [26], control of a football robot } \\
\text { team [12] }\end{array}$ \\
\hline Culture & $\begin{array}{l}\text { Remote tour guiding at a museum [69, 85, 15], interactive tour guide in museums } \\
{[114,15]}\end{array}$ \\
\hline Surveillance & $\begin{array}{l}\text { UAV [78, 79, 98], monitoring activities in factories, offices and industrial sites, remote } \\
\text { control [101, 98], detect internal condition of working areas [119], drones as a service } \\
\text { [65] }\end{array}$ \\
\hline Military & Warfare robot car [47], land mining and field surveillance $[8]$ \\
\hline Emergency/Disaster response & $\begin{array}{l}\text { Emergency response system [133, 42], drones for emergency use [65], crime situa- } \\
\text { tions [29] }\end{array}$ \\
\hline Rescue operations & UAV for object tracking [63], UAV for disaster rescue operations [2] \\
\hline Agriculture & $\begin{array}{l}\text { Soil moisture sensing and remote crop monitoring [125], live streaming of crops, seed } \\
\text { sowing, pesticide sprinkling and automatic irrigation [43] }\end{array}$ \\
\hline
\end{tabular}




\subsection{Hardware and Sensors}

\subsubsection{Hardware}

Besides the mechanical parts which vary significantly per related work under study, there is some common hardware used among the surveyed papers, such as Raspberry Pi [123, 11, 119, 26, 16, 101, 47, 63, 43] and Arduino [125, 101, 8]. Both Raspberry Pi and Arduino constitute open-source hardware and electronic prototyping platforms, enabling users to create interactive electronic objects. In the context of this survey, researchers in related work have used them as mini computers, connecting external sensors, actuators and mechanical parts, in order to give intelligence to their robots. TelosB was the sensor platform selected in [98].

Some efforts tried to develop humanoid robots [26, 104, 15, 114], while others employed autonomous vehicles [123, 34, 125], a space exploration rocket [10], a warfare car robot [47] and UAV/drones [78, 79, 65, 125, 133, 63, 2, 29, 98]. An UAV enhanced with IoT sensors has been introduced in [98], where the UAV interacts better with its environment towards more effective surveillance, gathering data coming from temperature, humidity and light sensors.

The remaining papers used robots with application-specific mechanics and characteristics. The interesting concept of biobots was introduced in [42], which was about real animals (in this case, dogs) equipped with sensors such as cameras or gas detectors. These sensors allowed the animals to sense additional environmental aspects (i.e. search rubble for casualties or detect dangers such as a gas leak). In this case, the mechanical parts of a robot are replaced by the physical capabilities of the animals involved.

\subsubsection{Sensors}

Robots were equipped with a wide variety of different sensors, such as RFID [14, 123, 124, 125, 44], video cameras [77, 96, 126, 34, 10, 88, 50, 112, 35], infrared and light sensors [77], smoke sensors [11], temperature and gas [119, 8], temperature, humidity and light sensors [98], medical sensors [6, 7, 44], accelerometers and gyros [41, 61], occupancy [23] and infrared sensors [43, 77, 96]. GPS receivers were also installed in many robots for localization and navigation [34, 125, 41, 65, 47, 63, 29].

The relationship between the aforementioned sensors and the robots' sensing/actuation capabilities, as they appear in related work, is presented in Table 3 . The relationship between the intended use of these sensors, the desired actions and targeted application areas is illustrated in Figure 5

Table 3: Sensors and intended use.

\begin{tabular}{|l|l|}
\hline Sensor & Intended Use \\
\hline Microphone & Voice recognition \\
\hline $\begin{array}{l}\text { Gyrometer, accelerome- } \\
\text { ter }\end{array}$ & Rotational motion \\
\hline RFID, Infrared sensor & Sense proximity \\
\hline $\begin{array}{l}\text { Occupancy, infrared sen- } \\
\text { sor }\end{array}$ & $\begin{array}{l}\text { Sense motion, perform some } \\
\text { mechanical action }\end{array}$ \\
\hline Video camera & $\begin{array}{l}\text { Computer vision, human remote } \\
\text { vision }\end{array}$ \\
\hline Pressure sensor & Sense pressure on something \\
\hline Medical sensors & $\begin{array}{l}\text { Measure health indications of } \\
\text { humans }\end{array}$ \\
\hline GPS receiver & Location \\
\hline Gas/chemical sensor & Sense hazardous materials \\
\hline Smoke sensor & Sense fire \\
\hline $\begin{array}{l}\text { Temperature/humidity } \\
\text { sensor }\end{array}$ & $\begin{array}{l}\text { Sense weather conditions, } \\
\text { surveillance }\end{array}$ \\
\hline Light sensor & $\begin{array}{l}\text { Measure illumination, surveil- } \\
\text { lance }\end{array}$ \\
\hline
\end{tabular}




\subsection{Software and Communications}

\subsubsection{Software}

In regards to software, some papers [63, 2, 29, 98] employed the popular Linux-based Robot Operating System (ROS) [90], which provides the communications infrastructure to program, operate, debug and control the robot as a system of systems.

ROS is an open-source framework for writing software for robotic systems. It consists of a number of libraries, tools and sets of conventions to simplify the task of writing software for complex mechatronic systems [90]. ROS includes a large variety of algorithms and functions for creating new software components and drivers. ROS supports multiple sensor technologies as well as programming languages, of which $\mathrm{C}++$ and Python are the most important. Nowadays, ROS is one of the most exploited tools for developing algorithms in the context of robotics due to its flexibility and simplicity. However, it is worth mentioning that ROS has no real-time capabilities. This means that ROS does not provide guarantees about the timing of operations, hence it is not intended for operations that have strict timing requirements.

In the case of safety critical systems in which hard real-time constraints exist, real-time operating systems must be adopted. The real-time operating system (RTOS) is one such system, used in [41], which handles the execution of tasks in order to meet their time deadline, but also facilitates memory management and accessing resources. The two main design philosophies are: event-driven and time sharing. An event-driven scheduler switches between tasks when an event of higher priority requires to be accommodated. On the other hand, a time sharing scheduler switches among tasks based on a periodic clock signal.

Other papers used GOLEX [15, 114], Embedded C [8], the Multi-target Robot Language (MRL) [77], Node.js [101] as well as OpenWSN [98]. OpenWSN is an open-source implementation of the IEEE/IETF 6TiSCH protocol stack [127]. 6TiSCH is a promising Working Group that aims to achieve industrial-grade performance in terms of jitter, latency, scalability, reliability and low-power operation for IPv6 over IEEE802.15.4e TSCH.

Besides the aforementioned software systems and platforms, Ray [91] has listed numerous emerging cloud-based robotics platforms that could be used together with IoRT architecture. As an example, Simoens et al. [104] employed the DYAMAND middleware [81] for abstracting the different protocols and interfaces of the installed sensors of the robot.

\subsubsection{Communication}

In terms of communication protocols, the majority of related work used Wi-Fi (i.e. 16 papers), while IEEE802.15.4 and ZigBee [47, 123, 124, 44, 98], 3G/4G [6, 7, 63] and Bluetooth [6, 7, 44, 104, 61, 47, 63] were also used. Regarding Bluetooth in particular, the Bluetooth Low Energy (BLE) technology was used for communication among robot components.

Wi-Fi has been used where wide coverage was required (i.e. up to 100 meters) and/or it was not possible to propagate messages via intermediate nodes [4]. Since Wi-Fi consumes much energy, it has been used where autonomy had not been an important issue (e.g. education, health, tour guiding in museums). 3G/4G has also been used in scenarios where connectivity was difficult (e.g. rescue operations) but it consumes more energy in comparison to Wi-Fi. On the other hand, ZigBee and BLE have been used for short-range and low-energy communication scenarios, where there was a specific (indoor) topology/infrastructure and need for autonomy (e.g. industry, domestic support, entertainment).

An aspect not really addressed in the surveyed papers is the security of software, communication messages and the actual data involved. Two papers mentioned the use of the HTTPS protocol for secure communication [6, 7] while NASA employed the WITS Encrypted Data Delivery System (WEDDS) and its public key infrastructure during the Mars polar lander mission [10].

\subsection{Summary}

It is worth investigating in which ways the IoT-enabled robots of the related work under study make actual use of Internet technologies, according to the definition of IoRT [91, 120, 105], as mentioned in the introduction. Table 4 presents the classification of the surveyed papers in different classes, based on how they use Internet technologies.

The majority of the surveyed papers use TCP/IP communications for interaction between the robot and the outside world [93]. Some of these papers incorporate principles of the WoT and they would be analyzed in the Section 5 . 
Table 4: Use of Internet technologies in IoRT.

\begin{tabular}{|c|c|}
\hline Type of use & Related Work \\
\hline Not any details & {$[61,78,79,2]$} \\
\hline IPv4 communication & $\begin{array}{l}{[125,96,11,119,10,6,5,26,111,16,41,88,44,104} \\
23,68,107,66,112,12,15,69, \\
35,314,\end{array}$ \\
\hline $\begin{array}{l}\text { Java Object Request Broker (ORB) architec- } \\
\text { ture }\end{array}$ & CORBA [50], HORB [71] \\
\hline IPv6 architectures & $\begin{array}{l}\text { 6LoWPAN [14], 6TiSCH technology (COAP, RPL, } \\
\text { 6LoWPAN) [98] }\end{array}$ \\
\hline Cloud robotics & {$[29,65,123,124,34,8,133,63,43,29]$} \\
\hline
\end{tabular}

Java ORB is used in [71, 50] for managing distributed program objects, while Brizzi et al. [14] employ an IPv6based 6LoWPAN architecture for communication between robots and wireless sensor networks. 6LoWPAN is a working group and standard for the application of IPv6 over low-power sensors and wireless sensor networks [80]. An implementation of 6LoWPAN via the 6TiSCH technology [127] was performed in [98], combining 6LoWPAN with relevant software protocols and implementations such as the Constrained Application Protocol (COAP) [100] and the RPL IPv6 routing protocol [130].

Finally, the last category of Table 4 is about papers using cloud services for storage, processing, updates and management/control. These papers touch upon the research area of cloud robotics [45], which deals with infrastructures and protocols for machine-to-cloud (M2C) communications. Some interesting relevant concepts mentioned in related work [34, 123] are Information Centric Networking (ICN) [1] and Software-Defined Networking (SDN) [74]. ICN is an approach to evolve the Internet infrastructure away from a host-centric paradigm based on the end-to-end principle, to a network architecture in which information is the focal point. SDN is an architecture that aims to make networks agile and flexible, improving network control. In the context of IoRT, ICN and SDN are approaches/architectures for facilitating robot control, as well as communication and networking between robots and the outside world through the Internet.

\section{Analysis of the Application of Web of Things in Robotics}

Table 5 lists which Web technologies have been used in the surveyed papers. Papers that do not use any Web technologies have been omitted from the table. Most of the papers use a basic HTTP interface for interacting with the robot, while the underlying communication is realized using different communication protocols. In Section 3.2. it was mentioned that WoT-based developments should employ REST and not only basic HTTP interfaces. However, we still include in Table 5 papers with only basic HTTP interfaces for interaction with robots, because they constitute the majority of related work.

Actual Web servers on the robot are installed in [41] (i.e. commercial Keil Tools $\mathrm{C} / \mathrm{C}++$ cloud-based compiler) and [101] (i.e. NodeJS server platform), while some papers move one step further, creating RESTful APIs for interacting with the robot's features and operations [119, 26, 63].

To enable Web-based interaction, some papers used platforms such as the WebIOPi IoT framework [119], the Web interface for telescience (WITS) [10], the WAMP server [26], the MASSIF platform [13, 104] and NodeJS [101]. These platforms provide Web servers and support for exposing robot services as API calls, control and debugging of the software used for programming the robots (e.g. WebIOPi for Raspberry Pi), control dashboards and easy installation setups (e.g. WAMP), remote access to the robot (e.g. WITS for the planetary rover mission to Mars [10]), management of asynchronous messaging and handling of thousands of concurrent connections (e.g. NodeJS), semantic annotation, reasoning and integration of IoT data (e.g. MASSIF [13]) etc.

Shin et al. [101] used the Web Service Description Language (WSDL) to describe the services provided by the REST API of their UAV [20]. WSDL [20], similar to Web Application Description Language (WADL) which is 
generally more suitable for Web-based applications [40], is used to describe service and their semantics in order for humans and machines to be able automatically to use these services by creating the appropriate requests via TCP/IP calls.

Finally, Web-based message brokers, such as RabbitMQ and Crossbar.io, are used in [104, 26]. These Web messaging brokers are suitable for high-performance, scalable distributed messaging where multiple publishers and subscribers of information are involved (e.g. health monitoring scenarios with care-providers involved [104, 26]).

Table 5: Use of Web technologies in WoRT.

\begin{tabular}{|c|c|}
\hline Type of use & Related Work \\
\hline $\begin{array}{l}\text { HTTP-based communica- } \\
\text { tion }\end{array}$ & {$[29,98]$} \\
\hline $\begin{array}{l}\text { Basic HTTP interface for } \\
\text { interacting with the robot }\end{array}$ & $\begin{array}{l}{[35,36,103,77,96,125,11} \\
10,6,5,50,107,15,69,114 \\
8,43,85]\end{array}$ \\
\hline Web server on the robot & [41, 101] \\
\hline $\begin{array}{l}\text { REST API for robot con- } \\
\text { trol }\end{array}$ & [119, 26, 63] \\
\hline $\begin{array}{l}\text { Semantic Web technolo- } \\
\text { gies }\end{array}$ & [104, 14] \\
\hline $\begin{array}{l}\text { Publish/Subscribe ar- } \\
\text { chitectures (Message } \\
\text { brokers) }\end{array}$ & [104, 26 \\
\hline
\end{tabular}

\subsection{Web Semantics}

An advanced aspect of WoT towards seamless M2M communication and understanding is the use of Semantic Web technologies to describe services and data, towards a semantic WoT [87]. The semantic WoT involves technologies for uniformly describing WoT data streams, devices and services, allowing easy and fast integration with other sources of information, platforms and applications towards advanced knowledge and reasoning [59].

Semantics are particularly important in robotics, for the understanding of space, ambient environment and surroundings of the robot and for better reasoning. Semantics can be described and analyzed by means of ontologies and vocabularies (e.g. OWL, SSN [22], IoT [99]). Ontologies include concepts and categories in a subject area or domain (i.e. where the robot is operating) that show, describe and explain their properties and the relations between them. Ontologies are not enough though and need to be accompanied by description languages (e.g. RDF, OWL), as well as query languages (e.g. SPARQL, CQEL). Description languages facilitate consistent encoding, exchange and processing of semantically-annotated content. The Resource Description Framework (RDF) is a general-purpose language for representing information on the Web, while the Web Ontology Language (OWL) is a formal language for representing ontologies in the Semantic Web. Semantic query languages are necessary for retrieving and manipulating data stored in description languages such as RDF/OWL, being able to answer complex queries and produce advanced knowledge by combining different sources of information together.

From the surveyed papers, Simoens et al. [104] employed semantic technologies to describe the robots' produced data by means of the WSN and SSN ontologies while Brizzi et al. [14] described robotic services by means of semantic web services. Semantic Web services [73] are similar to Web Services, but they additionally employ standards for the interchange of semantic data.

\section{Discussion}

This section discusses the general findings of the survey. Specifically, Section 6.1 refers to the technical aspects of the surveyed work and Section 6.2 examines the actual relationship between related work under study and the general 
principles of IoT/WoT. Then, Section 6.3 captures the big picture in relation to research and development in the area of robotic IoT, while Section 6.4 provides future research opportunities in the IoRT domain. Finally, Section 6.5 demonstrates our own IoT-enabled robot, used for inspection of pipelines and Section 6.6 summarizes the take-home messages of this survey paper.

\subsection{Technical Aspects}

A large percentage of the research work under study (36\%) employed open-source hardware and prototyping platforms, connecting them to a wide variety of sensors (i.e. 14 different sensor types). The mechanical parts of the robots involved 16 different actions, with movement, observation and navigation being the most popular ones. Sixteen application areas have been recorded, with health being the most popular application domain for IoT-based robotics. This makes sense, considering that health applications, especially at domestic level, require easy interconnection with other devices of a smart home or fast notification/alerting and communication with care providers. Thus, the IoT/WoT protocols are appropriate for this interconnection with low effort.

In regards to communications, Wi-Fi was the most popular technology (36\% of the papers), followed by Bluetooth $(16 \%)$ and ZigBee (10\%). The characteristics of these communication protocols are described in [4] and researchers should consider the most appropriate technology for their implementations, taking into account application requirements such as range and coverage, energy consumption and autonomy of the robot, security, mobility aspects etc.

Unfortunately, only 2 papers (4\%) explicitly mentioned security measures during message communication. Some papers might have used the security features provided by the underlying platforms and operating systems used (e.g. ROS, RTOS, MRL), but this has not been specified by the authors explicitly. We argue that nowadays IoT offers a wide range of secure communication protocols [82] and they should be harnessed by researchers to increase the security of their robot implementations. IoT could also be used to enhance humans' access control via smart authentication, i.e. by means of various biometrics, face or voice recognition etc.

\subsection{Connection to the Internet/Web of Things}

Our research shows that some papers claimed to be IoT-enabled without giving any details of the connection between IoT and robotics [61, 78, 79, 2]. Also, the majority of the surveyed papers (29 papers, 59\%) use merely TCP/IP communication, which is only a small part of the concepts of IoT and IoTR [91, 120, 105]. In regards to the WoT, many of the surveyed papers (20 papers, $40 \%$ ) perform HTTP-based communication or provide only some basic HTTP interface for interacting with the robots.

The most complete papers in IoRT are those employing cloud robotics (see Table 4, while the most complete ones in WoRT are those using REST APIs for robot control and/or semantic web technologies (see Table 55. The observations that a limited number of papers employ IPv6 architectures or cloud robotics (12 papers, 24\%) and/or use REST APIs or semantics (5 papers, 10\%) are indications that the penetration of IoT/WoT in robotics is still low and that the IoT/WoT are still not largely and properly used in robotics. This phenomenon has been observed also in WoT frameworks in the past [54], where the authors claimed to have WoT-ready frameworks, however their developments missed some important elements of the WoT principles.

As mentioned before, cloud networked robotics is a modern, promising research area [45]. New cloud-based software systems make the integration between robotics and IoT much easier [41, 116]. The ROS could be used for connecting robots to the cloud [90] (i.e. together with the FIROS tool [64]). Such possibilities are also provided by RoboEarth [122], a system for sharing knowledge between robots. Rapyuta, as the RoboEarth cloud engine, helps robots to offload heavy computation by providing secured customizable computing environments in the cloud [46]. Towards WoRT, the work in [60] allows to build REST APIs for ROS, while development of Web-based services for robotic devices is possible via [72].

\subsection{The Big Picture}

To complete the discussion on the IoRT/WoRT topics, we try to capture here the big picture of this research area.

First, it is important to mention the performance measures that can be used to assess the performance and/or compare various robot systems [108]. Many performance metrics exist in order to assess the quality of a robot or a network/swarm of robots, but the ones most commonly adopted are power/energy consumption, bandwidth, latency, throughput, resilience to errors, packet loss and locality of program execution. Some metrics are more suitable for 
UAV [52], such as setup time, flight time, inaccuracy of land, haptic control effort and coverage ratio. Unfortunately, our analysis cannot get in depth on this aspect because none of the cited authors discussed, mentioned or performed evaluation of the proposed robotic systems using these assessment metrics and/or and comparison with existing similar robot implementations. The only exception was the work in [98], where the authors assessed the quality of service of their robotic system (i.e. UAV with Telosb sensors), expressed in terms of network joining time, data retrieval delay and packet loss ratio. Their conclusion was that the performance of the UAV based on the aforementioned metrics satisfied the mission requirements.

Generally, authors of work under study preferred to focus their research on the feasibility and demonstration of connecting robots to the Internet/Web, as well as to the new possibilities (i.e. sensing, actions, application domains) that arose by their robot models/implementations (see Figure 5). We acknowledge the fact that in many cases there had not been any similar robotic systems to compare with.

It is still important to quantify and assess the impact of the TCP/IP stack and/or Web technologies to the real-time operations of robotic systems. The papers under study have not discussed this issue. Although other research works demonstrated that the impact of IoT/WoT on embedded devices is low [56, 48, 97, 27, 93], this might not be the case for time-critical robots.

Figure 6 depicts the general suggested architecture for IoRT/WoRT systems, considering the principles of IoT and WoT applied in robotics. Communication between sensors and electronic platforms is via the IPv6 protocol, while it is possible for sensory platforms to embed Web servers themselves [56]. Figure 6]lists some of the operating systems, as identified in related work under study for sensors' and robots' programming, or for deploying Web servers on the robots.

Some promising platforms for robots' programming not employed in the surveyed papers but still worth mentioning are FIROS [64], a tool for connecting mobile robots to the cloud (i.e. by using ROS), BrainOS1] an autonomous navigation platform based on computer vision and artificial intelligence, as well as the Middleware for Robotic Applications (MIRA) [28], a cross-platform framework for the development of robotic applications.

Towards full Web integration, robots may expose their services as a REST API, for easier reuse of their capabilities and features by authorized third parties. Robots may also incorporate semantic engines for annotating semantically their services and data, towards seamless M2M interaction. As mentioned in Section 5.1, researchers employed various ontologies to describe robotic data (i.e. WSN, SSN) and robotic services (i.e. Brizzi et al. [14]). A set of interesting ontologies that could be used in future IoRT systems is the IEEE Ontologies for Robotics and Automation (ORA) [89]. These ontologies could also describe the relationship between a robot agent and its physical environment. As an example, Jorge et al. [51] used the ORA ontology for spatial reasoning between two robots that must coordinate for providing a missing tool to a human. Another interesting set of semantics is proposed in [113], for projecting the effects of actions and processes performed by the robots and their sequence. Besides ontologies, query languages and engines (e.g. SPARQL, CQEL) are important for semantic reasoning.

For completing IoT integration, especially in cases where robot swarms are involved or high scalability/performance are needed, cloud services could be the solution. The cloud could be used for more advanced processing and for storage of information, but also for efficient messaging via publish/subscribe infrastructures. Concepts such as ICN [1] and SDN [74] could also be employed for better overall management and control of the robots in a more abstract and generic manner.

As mentioned in Section 2, comparison of IoT/WoT existing software and hardware platforms is out of the scope of this paper. It is worth commenting however that the inclusion of IoT/WoT protocols gives important benefits to (not only) robotic systems, such as interoperability, seamless M2M communication, easier integration to existing systems and infrastructures, as well as use of well-known and popular technologies for programming, management and control [129, 39]. Some of these benefits have been highlighted in Sections 3.1 and 3.2 As mentioned before however, more studies are needed in order to further validate the impact of the IoT/WoT protocols and technologies on the real-time performance of robots.

\subsection{Challenges and Future Opportunities}

Robotics, together with IoT, constitute dynamic and active research fields and there is much ongoing research in these areas. This section focuses on the existing challenges and barriers, as well as future research opportunities that

${ }^{1}$ BrainCorp, BrainOS. https://www.braincorp.com/brainos-autonomous-navigation-platform 


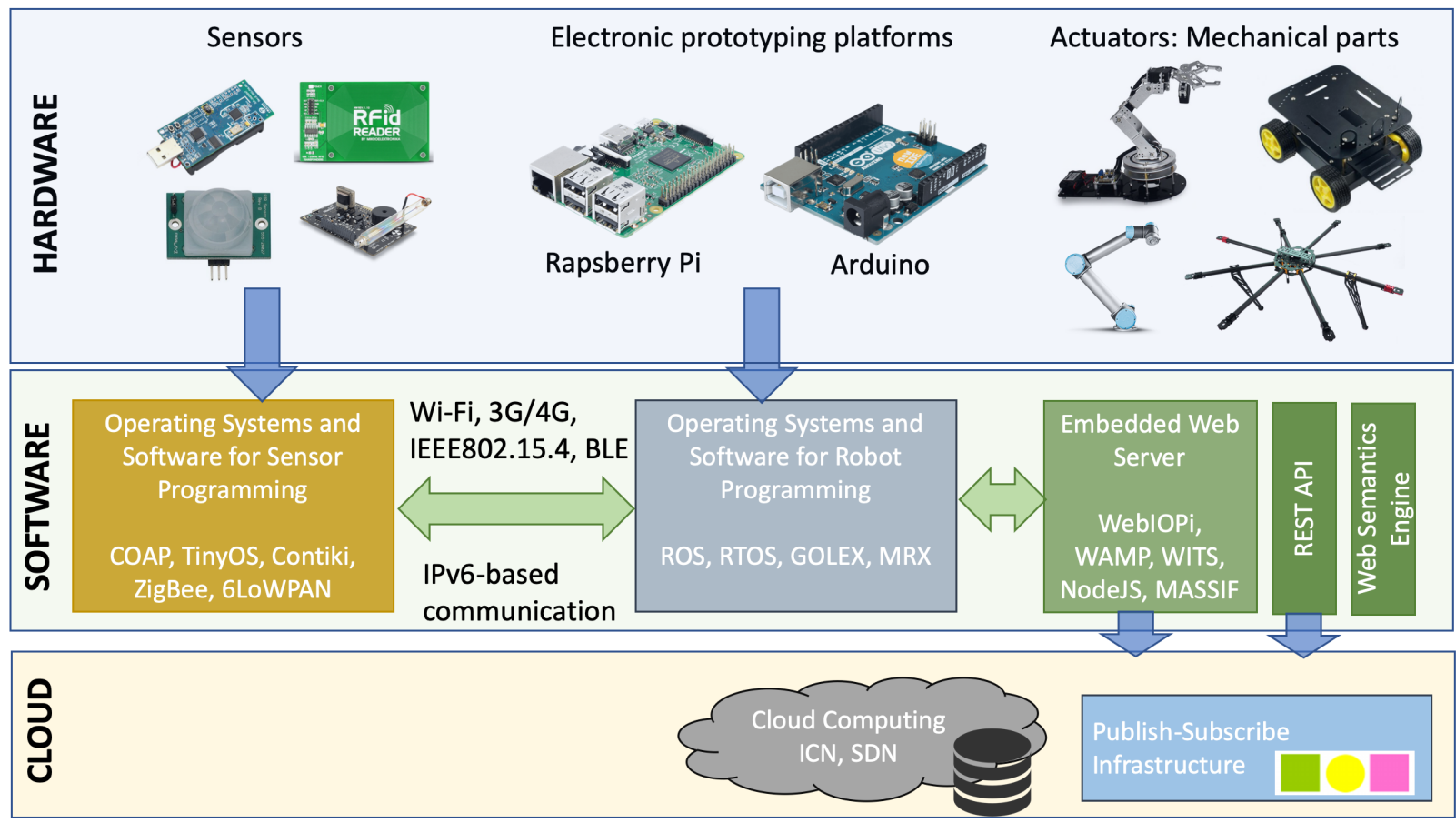

Figure 6: The general architecture for IoRT/WoRT systems.

arise from the combination of these technologies together in the future. Some of these challenges and/or opportunities are the following:

- Although the impact of IoT/WoT technologies and protocols on embedded devices may be low [56, 48, 97, 27, [93], this impact might still be considerable in time-critical robotic systems, where decisions need to be taken in fragments of a second. We welcome research efforts dealing with this aspect, studying in detail how different technologies and protocols of IoT and WoT affect performance. This could be well related to the security of the messages exchanged between robots and infrastructures [110].

- As mentioned in Section 6.3, it is unfortunate that no comparisons of the robots with existing literature in terms of performance metrics was performed in the related work under study. We expect that future research will focus more on performance, studying the possibilities and constraints of the robots considering low-level metrics such as energy consumption and autonomy, bandwidth, latency, throughput, resilience and fault tolerance, as well as high-level metrics such as ease of use and control of the robot, overall engagement and social behaviour, safety etc. It would be also important to study the penalty and trade-offs in energy consumption and autonomy when Web servers and the IPv6 stack are added to the robots [48, 56].

- In relation to the above, an important challenge( and desired characteristic) of robots is autonomy in operation. IoT has active research in breakthrough high-performance architectures, algorithms and hardware that will allow wireless networks to be highly efficient [70], powered by tiny batteries, energy-harvesting [53], or over-the-air power transfer. Moreover, new communication systems based on biology and chemistry are expected to evolve in IoT research, enabling a wide range of new micro- and macro-scale applications [3].

- Another important research challenge for robots is the collective behaviour of robot swarms, or the coordination and control of multi-robot systems towards an optimized outcome. Such swarms have been proposed for collective industrial construction [86], transportation and box pushing [84], as well as for smart homes [95] and search and rescue [24]. More generally, the authors in [67] propose a plan-based approach, based on PEIS Ecology [95], to automatically generate a preferred configuration of a robot ecology given a task, environment and set of resources. In this robot swarm ecosystem, IoT can offer effective solutions for networking, communication among the robots and mobility [24]. 
- Just like there exist complete operating systems (e.g. TinyOS, Contiki) and application-layer protocols (e.g. COAP, ZigBee) for IoT sensors and devices, we expect similar operating systems to appear for robots [131]. As mentioned before, early efforts in this direction are BrainOS and MIRA. A culture of developers and researchers still needs to be built around these platforms/frameworks, sharing code, experiences and solutions to the community. Worth mentioning is the Brains For Bots SDK by Neural $2^{2}$, which includes numerous features for creating applications that can learn, recognize, find and track objects in real-time. Neurala incorporates in its SDK deep learning techniques, to support cognitive requirements in robot applications.

- Combining online social networking together with IoT/WoT-enabled robots [117]. In this case, online social networks could be used for storing and sharing links to resources of interest for the R2R and R2H interactions, facilitating sharing of robotic services among online trustful contacts [57]. Robots could recognize or authenticate users, giving them access to some of their controls, based on the peoples' online profiles and endorsements they have from other authorized people.

- In industrial applications and logistics, technologies that blend the physical and digital context are important. Broader and more expansive information capture and processing via IoT/WoT, combined with smarter manipulation and movement of physical materials via robots, can deliver new benefits such as higher efficiency of operations, more insights and visibility, as well as better interaction between components, systems and actors. Blockchain could be relevant, to keep an immutable distributed ledger among IoT sensors and robotic systems of untrusted partners involved in some supply chain ecosystem [115, 120].

- The trend towards human-centric design of robotic systems is expected to continue [131] and robots will become more integrated in our everyday lives, either for assistance in common tasks or for actual support of people in need, e.g. movement of people with paralysis. Thus, they need to capture humans' emotions and social behaviour to understand how to react in different situations [31]. This information might come from IoT sensors, either installed on the robots or in the nearby environment (i.e. smart homes/buildings) or from the online social networking presence and activity of humans. Ethics is an important dimension in this direction [62] and needs to be considered a priori.

- Towards human-centric robotics, the teaching of robots to take actions by means of natural language instructions is a key aspect. This challenge could derive knowledge and ideas from Web Semantic technologies, as well as from ontology-based natural language interfaces for controlling IoT devices, such as im4Things [83] and home automation [9].

- We expect service robots to enter new real-world environments in which IoT has already penetrated [55]. These environments could be sports (i.e. assistance and safety in sports having risks such as climbing and parachuting), health (i.e. not only monitoring patients but also taking first-aid actions if needed), ecology and environmental monitoring, surveillance and security in social spaces (i.e. schools, airports, urban hotspots etc.), gaming (i.e. robots become comrades or opponents in real-world gaming scenarios), customer service (i.e. restaurants, hotels, gyms, tourism), the movie industry and many others. The autonomous learning capabilities of robots, together with their potential actions due to their mechanical parts, could offer more opportunities in these new environments.

\subsection{Demonstration of an IoT Robot for Pipeline Inspections}

A relevant application domain where robotics would be very useful is industrial inspection and maintenance, using autonomous robots. In the context of petrochemical pipeline plants, in-pipe inspection can be performed using snake-like robots [25].

The first steps in the direction of autonomous navigation and inspection have been taken at the Robotics and Mechatronics Department of the University of Twente. This is a new application in IoT-related robotics, according to Table 2. The process of crawling is also a new action according to Figure 5 , based on the capability of moving. Figure 7 presents the PIRATE (Pipeline Inspection Robot for AuTonomous Exploration) robot, which is designed to travel

\footnotetext{
${ }^{2}$ Neurala, Brains For Bots. https://www.welcome.ai/tech/hardware-iot/neurala-brains-for-bots-sdk
} 
through pipes having different diameters, vertical sections and sharp corners. The robot is composed of six modules connected through actuated rotational joints. Each module also includes a driven wheel.

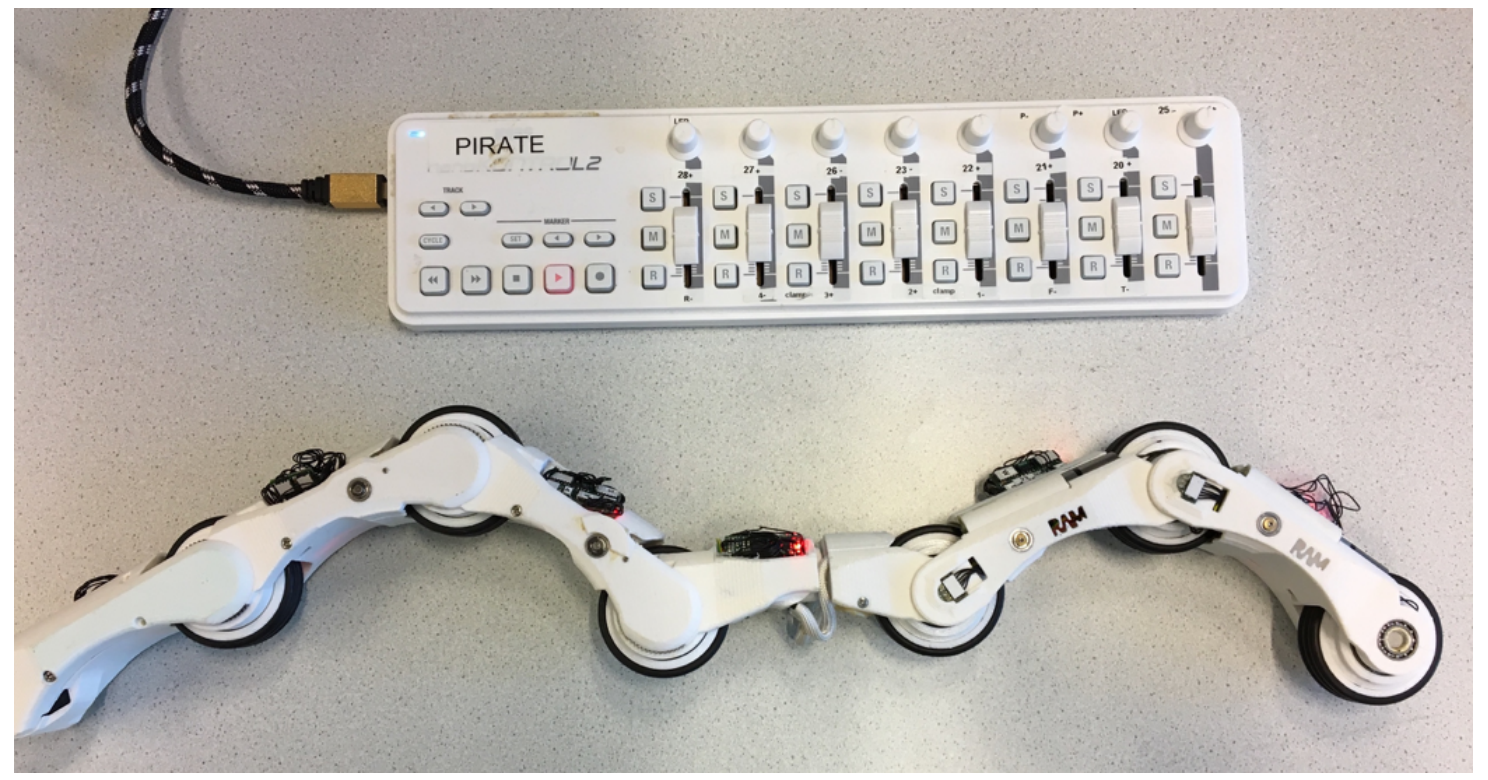

Figure 7: The PIRATE robot: It has a multi-links body in which each segment is coupled to the next one using an actuated rotational joint.

\subsubsection{Hardware}

PIRATE is equipped with absolute encoders for measuring the joints angles and consequently infer its configuration in the pipe and the wheels' speed, with inertial measurements units (IMU) for measuring the acceleration and orientation. Thanks to the encoders and the IMU, it is possible to infer the pose (i.e. position, joint configurations and orientation of the robot) in the pipe. This info is necessary for the autonomous control of the robot, especially for difficult moves such as taking a sharp 90 degrees turn, as shown in Figure 8

Moreover, the robot is enhanced with cameras for visual inspection, with Light Detection and Ranging (LiDAR) sensors for navigation and ultrasonic sensors for measuring the wall thickness. The latter is an important parameter for assessing the state of the pipe. LiDAR and ultrasonic constitute new sensing equipment for IoT robots, according to Figure 5 .

The robot is controlled via an Arduino board that is used as transparent bridge between the laptop which is used for programming and the robot and the (custom) boards on the robot. These boards directly control motors and read the information from the sensors. On each board, a microcontroller, a current regulator, an H-bridge, a RS485 driver and an IMU compass are installed. This configuration is depicted in Figure 9

The robot is currently wired (via an Ethernet cable), due to the fact that that popular wireless protocols did not function well inside pipe systems (e.g. IEEE802.15.3, Wi-Fi, BLE), according to our observations. Theoretically, super low frequency carrier wireless communication modules can be used [134], but we did not possess this equipment. It is an interesting aspect of future work.

\subsubsection{Software}

The software code has been developed on top of ROS [90], based on C++. ROS was selected because it offers great flexibility for designing software architectures, offering many libraries and algorithms for tasks such as motion, object detection, mapping, etc. (see Section 4.3.1). Its main disadvantage is that it does not support real-time guarantees, but this was not a requirement of our deployment.

An important characteristic of ROS is that it offers, thanks to FIROS [64], the possibility to connect robots and robotic services to the cloud. FIROS provides a publish-subscribe scheme and a REST API for managing robot connections and to receive the data published by the robot (i.e. publisher) via our laptop (i.e. subscriber). 


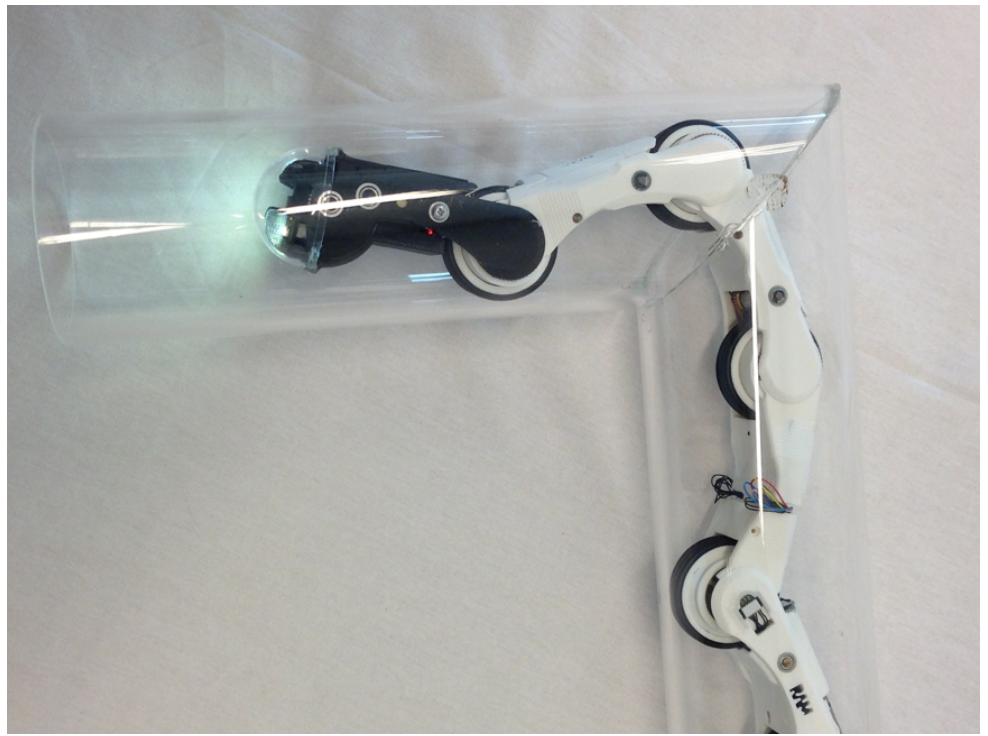

Figure 8: The PIRATE robot taking a sharp 90 degrees turn.
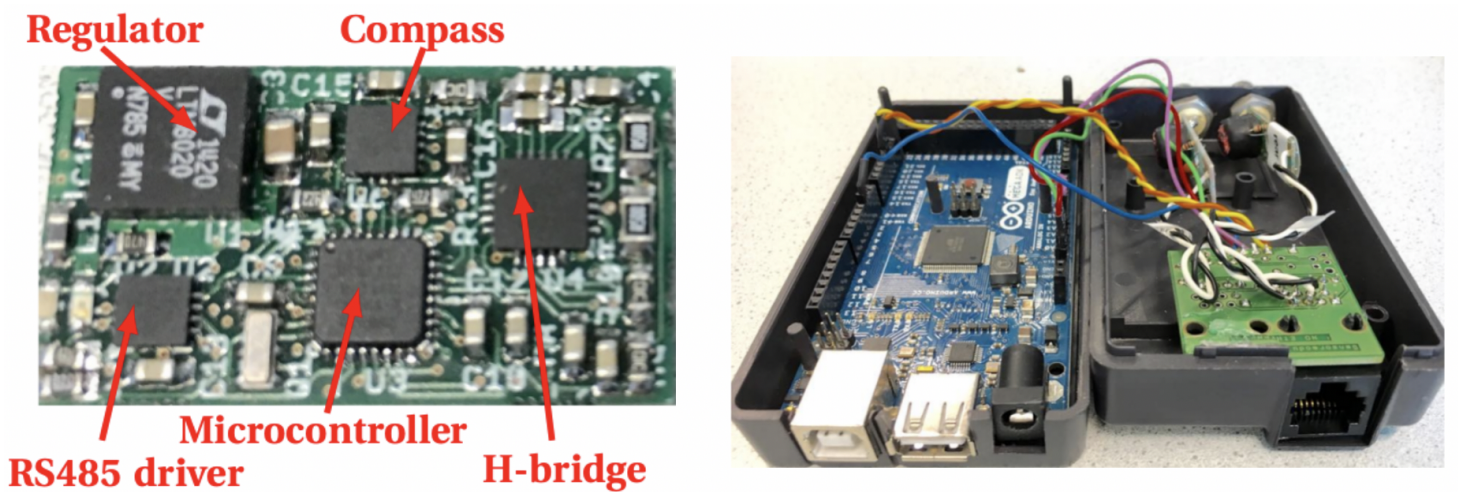

Figure 9: The control board (on the left) and the Arduino board (on the right) as used on the PIRATE robot.

Specifically, we used the following steps to enable PIRATE to the Internet/Web via FIROS:

1. First, we added the PIRATE robot to the whitelist of FIROS (i.e. a whitelist.json file) via the POST/whitelist/write API call, in order to make PIRATE discoverable and manageable.

2. Then, we used the POST/robot/connect API call to connect to the PIRATE robot. This call makes FIROS to connect to to new robots in case their names and topics match the ones allowed on the whitelist.json file.

3. Finally, the GET/robot/PIRATE API call was used to receive the robot's sensory information in a JSON format.

The performance of the robot in terms of task efficiency, reliability, sensor accuracy, response time and energy consumption constitutes subject of future work and it will be topic of another publication.

\subsubsection{Potential Benefits}

In addition to the general benefits of enabling robots to the IoT (see Section 3.1) and to the WoT (see Section 3.2 , in the context of the PIRATE robot this IoT/WoT enabling allowed the constant and continuous interaction and communication between the robot and human operators via a well-known and understood medium, i.e. the Internet. During the inspection mission, the robot gathered data about the environment (i.e. the pipe), which were described using semantic Web technologies via the SSN ontology [22]. Using this ontology (or other relevant ones), researchers 
may understand and reuse data collected by PIRATE without much effort and ambiguity. Using the Internet/Web as medium for gathering and storing data allows also to process and examine this data remotely and in real-time. Operators who may be located in different regions of the world may work together to indicate different inspection targets to PIRATE. Real-time Internet connectivity and data acquisition facilitates even the deployment of swarms of in-pipe robots, which may be deployed to increase speed and efficiency of the inspection procedure, while the communication provided by IoT protocols is crucial for the optimization of the task. The latter constitutes current work of the Robotics and Mechatronics Dept., aiming to develop IoT-enabled robotic swarms which allow real-time monitoring and control via the Internet/Web.

\subsection{Take-Home Messages}

Summarizing the study performed in this paper, some take-home messages can be the following:

- As the IoT penetrates different domains, application areas and scientific disciplines, it started to penetrate also the research area of robotic systems.

- There is a wide range of sensors used, intended actions of the robots and application areas where the IoRT/WoRTbased robots operate. The most popular actions involve moving, observing via computer vision, flying and navigating. The most popular application areas are entertainment, health, education and surveillance.

- A large percentage of the surveyed papers employed open-source hardware and prototyping platforms, making the physical connection to a wide variety of different sensors possible.

- Regarding communications, various protocols are being used, with Wi-Fi being the most popular, followed by IEEE802.15.4 and Bluetooth. The decision on which communication protocol to use was mainly influenced by the coverage range required, as well as the need for autonomy and longer lifetime of operation (i.e. energy consumption).

- The IoT is still not used at its full potential in robotics. Cloud robotics and the IPv6 protocol are not fully utilized. The same holds for the principles of WoT, which has not yet been fully exploited in robotic systems. Services of the robots are not generally exposed as a REST API, nor the services and data are described via Web semantic technologies.

- There is an increasing number of operating systems, software tools and platforms for developing IoRT/WoRTbased robotic systems. Most of this software enables access to cloud computing and publish-subscribe infrastructures for scaling computation and storage capabilities and for supporting robot swarms more easily.

- Related work mostly focuses on feasibility studies and demonstrations, which showcase the benefits of IoT/WoT in robotics, mainly in terms of ubiquitous access via Internet/Web and easy interoperability with other systems. Aspects of performance, especially considering the overhead produced by the TCP/IP protocol, have not been well studied.

- Although security and privacy constitute important aspects of robots and their services/data, they have not been addressed by the works under study.

- There are still numerous open issues and gaps for future research in this emerging intersection of research areas. Important research directions relate to the presence of robots in online social networks, autonomy in operation, collective behaviour of robot swarms, taking actions by means of natural language, deriving knowledge from Web semantics, design of more empathic, social, human-centred service-based robots and others.

\section{Conclusion}

This paper studied the current use of the Internet of Things (IoT) in robotics, through various real-world examples encountered through a research based on a bibliographic-based method. The concepts, characteristics and architectures of IoT, as they are being used in existing robotic systems, have been recorded and listed, together with popular 
software, hardware and communication methods. Moreover, the application areas, sensors and robot services/actions incorporated in IoT-based robots are presented. Further, the current application of the Web of Things (WoT) in robotics has been investigated and the overall potential of the Web of Robotic Things has been discussed in the paper. A general observation is that some of the advanced concepts of IoT/WoT are not yet being used by researchers in robotics. Finally, future research directions and opportunities are proposed.

\section{Acknowledgements}

Andreas Kamilaris has received funding from the European Union's Horizon 2020 research and innovation programme under grant agreement No 739578 complemented by the Government of the Republic of Cyprus through the Directorate General for European Programmes, Coordination and Development.

Nicolò Botteghi has received funding from Smart Tooling. Smart Tooling is an Interreg Flanders-Netherlands project sponsored by the European Union focused on automation in the process industry: making maintenance safer, cheaper, cleaner, and more efficient by developing new robot prototypes and tools.

\section{References}

[1] Bengt Ahlgren, Christian Dannewitz, Claudio Imbrenda, Dirk Kutscher, and Borje Ohlman. A survey of information-centric networking. IEEE Communications Magazine, 50(7), 2012.

[2] Taemin Ahn, Jihoon Seok, Inbok Lee, and Junghee Han. Reliable Flying IoT Networks for UAV Disaster Rescue Operations. Mobile Information Systems, 2018, 2018.

[3] Ian F Akyildiz and Josep Miquel Jornet. The internet of nano-things. IEEE Wireless Communications, 17(6):58-63, 2010.

[4] Shadi Al-Sarawi, Mohammed Anbar, Kamal Alieyan, and Mahmood Alzubaidi. Internet of things (iot) communication protocols. In 8th International Conference on Information Technology (ICIT), pages 685-690. IEEE, 2017.

[5] Majid A Al-Taee, Ala H Sungoor, Suhail N Abood, and Nada Y Philip. Web-of-Things inspired e-Health platform for integrated diabetes care management. In Applied Electrical Engineering and Computing Technologies (AEECT), IEEE Jordan Conference on, pages 1-6, 2013.

[6] Majid A Al-Taee, Waleed Al-Nuaimy, Ali Al-Ataby, Zahra J Muhsin, and Suhail N Abood. Mobile health platform for diabetes management based on the Internet-of-Things. In Applied Electrical Engineering and Computing Technologies (AEECT), IEEE Jordan Conference on, pages 1-5, 2015

[7] Majid A Al-Taee, Waleed Al-Nuaimy, Zahra J Muhsin, and Ali Al-Ataby. Robot assistant in management of diabetes in children based on the Internet of things. interaction, 11:13, 2017.

[8] M. Ashokkumar and T. Thirumurugan. Integrated IOT based design and Android operated Multi-purpose Field Surveillance Robot for Military Use. Advances in Engineering Research (AER), 142(1):236-243, 2018.

[9] Cyril Joe Baby, Faizan Ayyub Khan, and JN Swathi. Home automation using iot and a chatbot using natural language processing. In Innovations in Power and Advanced Computing Technologies (i-PACT), pages 1-6. IEEE, 2017.

[10] Paul G Backes, Kam S Tso, Jeffrey S Norris, Gregory K Tharp, Jeffrey T Slostad, Robert G Bonitz, and Khaled S Ali. Internet-based operations for the Mars polar lander mission. In Robotics and Automation (ICRA), IEEE International Conference on, volume 2, pages 2025-2032. IEEE, 2000.

[11] Sunil Bhutada, L Sai Tejasri, and R Tejaswini. IoT based Mobile Robot for Smoke Detection. International Research Journal of Engineering and Technology (IRJET), 5(4):1381-1383, 2018.

[12] Zhuming Bi, Guoping Wang, Li Da Xu, Matt Thompson, Raihan Mir, Jeremy Nyikos, Armela Mane, Colton Witte, and Cliff Sidwell. IoT-based system for communication and coordination of football robot team. Internet Research, 27(2):162-181, 2017.

[13] Pieter Bonte, Femke Ongenae, Femke De Backere, Jeroen Schaballie, Dörthe Arndt, Stijn Verstichel, Erik Mannens, Rik Van de Walle, and Filip De Turck. The massif platform: a modular and semantic platform for the development of flexible iot services. Knowledge and Information Systems, 51(1):89-126, 2017.

[14] Paolo Brizzi, Davide Conzon, Hussein Khaleel, Riccardo Tomasi, Claudio Pastrone, AM Spirito, M Knechtel, Ferry Pramudianto, and P Cultrona. Bringing the Internet of Things along the manufacturing line: A case study in controlling industrial robot and monitoring energy consumption remotely. In Emerging Technologies and Factory Automation (ETFA), 18th Conference on, pages 1-8. IEEE, 2013.

[15] Wolfram Burgard, Armin B Cremers, Dieter Fox, Dirk H'ahnel, Gerhard Lakemeyer, Dirk Schulz, Walter Steiner, and Sebastian Thrun. Experiences with an interactive museum tour-guide robot. Artificial intelligence, 114(1-2):3-55, 1999.

[16] Victor Callaghan. Buzz-Boarding; practical support for teaching computing based on the internet-of-things. In 1st Annual Conference on the Aiming for Excellence in STEM Learning and Teaching, Imperial College, London and The Royal Geographical Society, pages 12-13, 2012

[17] Marco Casini. Internet of things for energy efficiency of buildings. International Scientific Journal Architecture and Engineering, 2(1): 24-28, 2014.

[18] M Cenk cCavucsouglu, Winthrop Williams, Frank Tendick, and S Shankar Sastry. Robotics for telesurgery: Second generation Berkeley/UCSF laparoscopic telesurgical workstation and looking towards the future applications. Industrial Robot: An International Journal, 30 (1):22-29, 2003.

[19] Thang Vu Chien, Hung Nguyen Chan, and Thanh Nguyen Huu. A comparative study on operating system for wireless sensor networks. In International Conference on Advanced Computer Science and Information Systems, pages 73-78. IEEE, 2011. 
[20] Erik Christensen, Francisco Curbera, Greg Meredith, Sanjiva Weerawarana, et al. Web services description language (wsdl) 1.1, 2001.

[21] Steven E Collier. The emerging enernet: Convergence of the smart grid with the internet of things. IEEE Industry Applications Magazine, 23(2):12-16, 2016.

[22] Michael Compton, Payam Barnaghi, Luis Bermudez, Ra'uL Garc'iA-Castro, Oscar Corcho, Simon Cox, John Graybeal, Manfred Hauswirth, Cory Henson, Arthur Herzog, et al. The ssn ontology of the w3c semantic sensor network incubator group. Web semantics: science, services and agents on the World Wide Web, 17:25-32, 2012.

[23] Silvia Coradeschi, Amadeo Cesta, Gabriella Cortellessa, Luca Coraci, Javier Gonzalez, Lars Karlsson, Francesco Furfari, Amy Loutfi, Andrea Orlandini, Filippo Palumbo, et al. Giraffplus: Combining social interaction and long term monitoring for promoting independent living. In Human system interaction (HSI), 6th International Conference on, pages 578-585. IEEE, 2013.

[24] Saumitra M Das, Y Charlie Hu, CS George Lee, and Yung-Hsiang Lu. Mobility-aware ad hoc routing protocols for networking mobile robot teams. Journal of communications and networks, 9(3):296-311, 2007.

[25] Edwin Christian Dertien. Design of an inspection robot for small diameter gas distribution mains. University of Twente, 2014.

[26] Panagiotis Doxopoulos, Konstantinos L Panayiotou, Emmanouil G Tsardoulias, and Andreas L Symeonidis. Creating an extrovert robotic assistant via IoT networking devices. arXiv preprint arXiv:1804.04361, 2018.

[27] Dunkels, Adam and others. Efficient application integration in ip-based sensor networks. In Proceedings of the First ACM Workshop on Embedded Sensing Systems for Energy-Efficiency in Buildings, pages 43-48. ACM, 2009.

[28] Erik Einhorn, Tim Langner, Ronny Stricker, Christian Martin, and Horst-Michael Gross. Mira-middleware for robotic applications. In IEEE/RSJ International Conference on Intelligent Robots and Systems, pages 2591-2598, 2012.

[29] Gabriele Ermacora, Antonio Toma, Basilio Bona, Marcello Chiaberge, Mario Silvagni, Marco Gaspardone, and Roberto Antonini. A cloud robotics architecture for an emergency management and monitoring service in a smart city environment. Polytech. Univ. Turin, Turin, Italy, Tech. Rep, 2013.

[30] Xinyang Feng, Jianjing Shen, and Ying Fan. Rest: An alternative to rpc for web services architecture. In First International Conference on Future Information Networks, pages 7-10. IEEE, 2009.

[31] Terrence Fong, Illah Nourbakhsh, and Kerstin Dautenhahn. A survey of socially interactive robots. Robotics and autonomous systems, 42 (3-4):143-166, 2003.

[32] James L Fuller. Robotics. Prentice Hall PTR, 1998.

[33] Padmini Gaur and Mohit P Tahiliani. Operating systems for iot devices: A critical survey. In IEEE Region 10 Symposium, pages 33-36. IEEE, 2015.

[34] Mario Gerla, Eun-Kyu Lee, Giovanni Pau, and Uichin Lee. Internet of vehicles: From intelligent grid to autonomous cars and vehicular clouds. In Internet of Things (WF-IoT), IEEE World Forum on, pages 241-246, 2014.

[35] Ken Goldberg, Michael Mascha, Steve Gentner, Nick Rothenberg, Carl Sutter, and Jeff Wiegley. Desktop teleoperation via the world wide web. In Proceedings of 1995 IEEE International Conference on Robotics and Automation, volume 1, pages 654-659. IEEE, 1995.

[36] Ken Goldberg, Joseph Santarromana, George Bekey, Steven Gentner, Rosemary Morris, Jeff Wiegley, and Erich Berger. The telegarden. In Proc. of ACM SIGGRAPH, pages 135-1140, 1995

[37] Luigi Alfredo Grieco, Alessandro Rizzo, Simona Colucci, Sabrina Sicari, Giuseppe Piro, Donato Di Paola, and Gennaro Boggia. Iot-aided robotics applications: Technological implications, target domains and open issues. Computer Communications, 54:32-47, 2014.

[38] Dominique Guinard, Vlad Trifa, Thomas Pham, and Olivier Liechti. Towards physical mashups in the web of things. In Networked Sensing Systems (INSS), Sixth International Conference on, pages 1-4. IEEE, 2009.

[39] Dominique Guinard, Vlad Trifa, Friedemann Mattern, and Erik Wilde. From the internet of things to the web of things: Resource-oriented architecture and best practices. In Architecting the Internet of things, pages 97-129. Springer, 2011.

[40] Marc J Hadley. Web application description language (wadl). 2006.

[41] James O Hamblen and Gijsbert ME Van Bekkum. An Embedded Systems Laboratory to Support Rapid Prototyping of Robotics and the Internet of Things. IEEE Trans. Education, 56(1):121-128, 2013.

[42] Tam Harbert. On the Internet of Things, Nobody Knows Youre a Dog, 2014. http://spectrum.ieee.org/computing/embedded-systems/on-theinternet-of-things-nobody-knows-youre-a-dog.

[43] P. Hemalatha, K. Dhanalakshmi, S. Matilda, and M.B. Anand. Farmbot-a Smart Agriculture Assistor Using Internet of Things. International Journal of Pure and Applied Mathematics, 119(10):557-566, 2018.

[44] Fang Hu, Dan Xie, and Shaowu Shen. On the application of the internet of things in the field of medical and health care. In International Conference on Cyber, Physical and Social Computing, pages 2053-2058. IEEE, 2013.

[45] Guoqiang Hu, Wee Peng Tay, and Yonggang Wen. Cloud robotics: architecture, challenges and applications. IEEE Network, $26(3), 2012$.

[46] Dominique Hunziker, Mohanarajah Gajamohan, Markus Waibel, and Raffaello D'Andrea. Rapyuta: The roboearth cloud engine. In ICRA, pages 438-444. Citeseer, 2013.

[47] Laiqa Binte Imran, Muhammad Farhan, Rana M Amir Latif, and Ahsan Rafiq. Design of an IoT based warfare car robot using sensor network connectivity. In 2nd International Conference on Future Networks and Distributed Systems, page 55. ACM, 2018.

[48] Antonio J Jara, Miguel A Zamora, and Antonio Skarmeta. Glowbal IP: An adaptive and transparent IPv6 integration in the Internet of Things. Mobile Information Systems, 8(3):177-197, 2012.

[49] Zhanlin Ji, Ivan Ganchev, M'airt'in O'Droma, Li Zhao, and Xueji Zhang. A cloud-based car parking middleware for IoT-based smart cities: Design and implementation. Sensors, 14(12):22372-22393, 2014.

[50] Songmin Jia and Kunikatsu Takase. Internet-based robotic system using CORBA as communication architecture. Journal of Intelligent and Robotic Systems, 34(2):121-134, 2002.

[51] Vitor AM Jorge, Vitor F Rey, Renan Maffei, Sandro Rama Fiorini, Joel Luis Carbonera, Flora Branchi, Joao P Meireles, Guilherme S Franco, Flavia Farina, Tatiana S Da Silva, et al. Exploring the ieee ontology for robotics and automation for heterogeneous agent interaction. Robotics and Computer-Integrated Manufacturing, 33:12-20, 2015.

[52] Chanyoung Ju and Hyoung Son. Multiple uav systems for agricultural applications: control, implementation, and evaluation. Electronics, 7 (9):162, 2018. 
[53] Pouya Kamalinejad, Chinmaya Mahapatra, Zhengguo Sheng, Shahriar Mirabbasi, Victor CM Leung, and Yong Liang Guan. Wireless energy harvesting for the internet of things. IEEE Communications Magazine, 53(6):102-108, 2015.

[54] Andreas Kamilaris and Muhammad Intizar Ali. Do Web of Things platforms truly follow the Web of Things? In Internet of Things (WF-IoT), IEEE 3rd World Forum on, pages 496-501, 2016.

[55] Andreas Kamilaris and Andreas Pitsillides. Mobile Phone Computing and the Internet of Things: A Survey. IEEE Internet of Things (IoT) Journal, 3(6):885-898, December 2016.

[56] Andreas Kamilaris, Vlad Trifa, and Andreas Pitsillides. Homeweb: An application framework for web-based smart homes. In 18th International Conference on Telecommunications, pages 134-139. IEEE, 2011.

[57] Andreas Kamilaris, George Taliadoros, Andreas Pitsillides, and Diomidis Papadiomidous. The practice of online social networking of the physical world. International Journal of Space-Based and Situated Computing, 2(4):240-252, 2012.

[58] Andreas Kamilaris, Feng Gao, Francesc X. Prenafeta-Bold, and Muhammad Intizar Ali. Agri-IoT: A Semantic Framework for Internet of Things-enabled Smart Farming Applications. In IEEE World Forum on Internet of Things (WF-IoT), Reston, VA, USA, December 2016. IEEE.

[59] Andreas Kamilaris, Andreas Pitsillides, Francesc X. Prenafeta-Bold, and Muhammad Intizar Al. A Web of Things Based Eco-System for Urban Computing - Towards Smarter Cities. In 24th International Conference on Telecommunications (ICT), Limassol, Cyprus, May 2017.

[60] Felix Leif Keppmann, Maria Maleshkova, and Andreas Harth. Building rest apis for the robot operating system-mapping concepts, interaction. In SALAD@ESWC, pages 10-19, 2015.

[61] Min Su Kim, Jae Geun Lee, and Soon Ju Kang. A Self-Organizing Interaction and Synchronization Method between a Wearable Device and Mobile Robot. Sensors, 16(6):842, 2016.

[62] Tobias Körtner. Ethical challenges in the use of social service robots for elderly people. Zeitschrift für Gerontologie und Geriatrie, 49(4): 303-307, 2016.

[63] Anis Koubya and B Quershi. Dronetrack: Cloud-based real-time object tracking using unmanned aerial vehicles. IEEE Access, 2018.

[64] Raffaele Limosani, Alessandro Manzi, Laura Fiorini, Paolo Dario, and Filippo Cavallo. Connecting ROS and FIWARE: Concepts and Tutorial. In Robot Operating System (ROS), pages 449-475. Springer, 2019.

[65] Seng W Loke. The internet of flying-things: Opportunities and challenges with airborne fog computing and mobile cloud in the clouds. arXiv preprint arXiv:1507.04492, 2015.

[66] Xiao Lu, Wenjun Liu, Haixia Wang, and Qia Sun. Robot control design based on smartphone. In Control and Decision Conference (CCDC), 25th Chinese, pages 2820-2823. IEEE, 2013.

[67] Robert Lundh, Lars Karlsson, and Alessandro Saffiotti. Dynamic self-configuration of an ecology of robots. In IEEE/RSJ International Conference on Intelligent Robots and Systems, pages 3403-3409, 2007.

[68] Ren C Luo, Kuo L Su, Jyh Hwa Tzou, and SH Henry Phang. Multisensor based control of pet robot through the Internet. In Industrial Electronics Society (IECON), 27th Annual Conference of the, volume 1, pages 416-421. IEEE, 2001.

[69] Shoichi Maeyama, S Yuta, and Akira Harada. Remote viewing on the Web using multiple mobile robotic avatars. In Intelligent Robots and Systems, IEEE/RSJ International Conference on, volume 2, pages 637-642, 2001.

[70] Mahmoud S Mahmoud and Auday AH Mohamad. A study of efficient power consumption wireless communication techniques/modules for internet of things (iot) applications. 2016.

[71] Kohji Masuda, Eizen Kimura, Norihiko Tateishi, and Ken Ishihara. Three dimensional motion mechanism of ultrasound probe and its application for tele-echography system. In Intelligent Robots and Systems, IEEE/RSJ International Conference on, volume 2, pages 11121116. IEEE, 2001.

[72] Simon Mayer. Web-based service brokerage for robotic devices. In ACM Conference on Ubiquitous Computing, pages 863-865, 2012.

[73] Sheila A McIlraith, Tran Cao Son, and Honglei Zeng. Semantic web services. IEEE intelligent systems, 16(2):46-53, 2001.

[74] Nick McKeown. Software-defined networking. INFOCOM keynote talk, 17(2):30-32, 2009.

[75] Julien Mineraud, Oleksiy Mazhelis, Xiang Su, and Sasu Tarkoma. A gap analysis of internet-of-things platforms. Computer Communications, 89:5-16, 2016.

[76] Daniele Miorandi, Sabrina Sicari, Francesco De Pellegrini, and Imrich Chlamtac. Internet of things: Vision, applications and research challenges. Ad hoc networks, 10(7):1497-1516, 2012.

[77] Fumio Mizoguchi, Hironori Hiraishi, and Hiroyuk1 Nishiyama. Human-robot collaboration in the smart office environment. In Advanced Intelligent Mechatronics, IEEE/ASME International Conference on, pages 79-84. IEEE, 1999.

[78] Mohammad Mozaffari, Walid Saad, Mehdi Bennis, and M'erouane Debbah. Mobile Internet of Things: Can UAVs provide an energyefficient mobile architecture? In Global Communications Conference (GLOBECOM), pages 1-6. IEEE, 2016.

[79] Mohammad Mozaffari, Walid Saad, Mehdi Bennis, and M'erouane Debbah. Mobile unmanned aerial vehicles (UAVs) for energy-efficient internet of things communications. IEEE Transactions on Wireless Communications, 16(11):7574-7589, 2017.

[80] Geoff Mulligan. The 6lowpan architecture. In Proceedings of the 4th workshop on Embedded networked sensors, pages 78-82. ACM, 2007.

[81] Jelle Nelis, Tom Verschueren, Dieter Verslype, and Chris Develder. Dyamand: dynamic, adaptive management of networks and devices. In 37th Annual IEEE Conference on Local Computer Networks, pages 192-195, 2012.

[82] Kim Thuat Nguyen, Maryline Laurent, and Nouha Oualha. Survey on secure communication protocols for the internet of things. Ad Hoc Networks, 32:17-31, 2015.

[83] José Ángel Noguera-Arnaldos, Mario Andrés Paredes-Valverde, María Pilar Salas-Zárate, Miguel Ángel Rodríguez-García, Rafael ValenciaGarcía, and José Luis Ochoa. im4things: an ontology-based natural language interface for controlling devices in the internet of things. In Current Trends on Knowledge-Based Systems, pages 3-22. Springer, 2017.

[84] Lynne E Parker and Fang Tang. Building multirobot coalitions through automated task solution synthesis. Proceedings of the IEEE, 94(7): 1289-1305, 2006.

[85] Eric Paulos and John Canny. Delivering real reality to the world wide web via telerobotics. In Robotics and Automation, IEEE International Conference on, volume 2, pages 1694-1699, 1996.

[86] Kirstin Hagelskjaer Petersen, Radhika Nagpal, and Justin K Werfel. Termes: An autonomous robotic system for three-dimensional collective 
construction. 2011.

[87] Dennis Pfisterer, Kay Romer, Daniel Bimschas, Oliver Kleine, Richard Mietz, Cuong Truong, Henning Hasemann, Alexander Kr"oller, Max Pagel, Manfred Hauswirth, et al. Spitfire: toward a semantic web of things. IEEE Communications Magazine, 49(11):40-48, 2011.

[88] Ignas Plauska and Robertas Damavsevivcius. Educational robots for Internet-of-Things supported collaborative learning. In International Conference on Information and Software Technologies, pages 346-358. Springer, 2014.

[89] Edson Prestes, Joel Luis Carbonera, Sandro Rama Fiorini, Vitor AM Jorge, Mara Abel, Raj Madhavan, Angela Locoro, Paulo Goncalves, Marcos E Barreto, Maki Habib, et al. Towards a core ontology for robotics and automation. Robotics and Autonomous Systems, 61(11): 1193-1204, 2013.

[90] Morgan Quigley, Ken Conley, Brian Gerkey, Josh Faust, Tully Foote, Jeremy Leibs, Rob Wheeler, and Andrew Y Ng. ROS: an open-source Robot Operating System. In ICRA workshop on open source software, volume 3, Kobe, Japan, 2009.

[91] Partha Pratim Ray. Internet of Robotic Things: Concept, Technologies, and Challenges. IEEE Access, 4:9489-9500, 2016.

[92] Cristanel Razafimandimby, Valeria Loscri, and Anna Maria Vegni. A neural network and iot based scheme for performance assessment in internet of robotic things. In 2016 IEEE first international conference on internet-of-things design and implementation (IoTDI), pages 241-246. IEEE, 2016.

[93] Joel JPC Rodrigues and Paulo ACS Neves. A survey on ip-based wireless sensor network solutions. International Journal of Communication Systems, 23(8):963-981, 2010

[94] Ankur Roy Chowdhury. Iot and robotics: a synergy. PeerJ Preprints, 5:e2760v1, 2017.

[95] Alessandro Saffiotti and Mathias Broxvall. Peis ecologies: Ambient intelligence meets autonomous robotics. In Proceedings of the Joint Conference on Smart Objects and Ambient Intelligence: Innovative Context-Aware Services: Usages and Technologies, pages $277-281$. ACM, 2005.

[96] Patrick Saucy and Francesco Mondada. Khepontheweb: open access to a mobile robot on the internet. IEEE robotics $\mathcal{G}$ automation magazine, 7(1):41-47, 2000.

[97] Lars Schor, Philipp Sommer, and Roger Wattenhofer. Towards a zero-configuration wireless sensor network architecture for smart buildings. In First ACM Workshop on Embedded Sensing Systems for Energy-Efficiency in Buildings, pages 31-36, 2009.

[98] Vito Scilimati, Antonio Petitti, Pietro Boccadoro, Roberto Colella, Donato Di Paola, Annalisa Milella, and Luigi Alfredo Grieco. Industrial internet of things at work: a case study analysis in robotic-aided environmental monitoring. IET wireless sensor systems, 7(5):155-162, 2017.

[99] Nicolas Seydoux, Khalil Drira, Nathalie Hernandez, and Thierry Monteil. Iot-o, a core-domain iot ontology to represent connected devices networks. In European Knowledge Acquisition Workshop, pages 561-576. Springer, 2016.

[100] Zach Shelby, Klaus Hartke, and Carsten Bormann. The constrained application protocol (coap). Technical report, 2014.

[101] Moon Sun Shin, Byung Cheol Kim, Seon Min Hwang, and Myeong Cheol Ko. Design and Implementation of IoT-based Intelligent Surveillance Robot. Studies in Informatics and Control, 25(4):422, 2016.

[102] Bruno Siciliano, Lorenzo Sciavicco, Luigi Villani, and Giuseppe Oriolo. Robotics: modelling, planning and control. Springer Science and Business Media, 2010

[103] Reid Simmons, Richard Goodwin, Sven Koenig, Joseph OSullivan, and Greg Armstrong. Xavier: An autonomous mobile robot on the web. Beyond Webcams: an introduction to online robots, page 81, 2002.

[104] Pieter Simoens, Christof Mahieu, Femke Ongenae, Femke De Backere, Stijn De Pestel, Jelle Nelis, Filip De Turck, Shirley A Elprama, Katriina Kilpi, Charlotte Jewell, et al. Internet of robotic things: Context-aware and personalized interventions of assistive social robots. In 5th IEEE International Conference on Cloud Networking (Cloudnet), pages 204-207, 2016.

[105] Pieter Simoens, Mauro Dragone, and Alessandro Saffiotti. The Internet of Robotic Things: A review of concept, added value and applications. International Journal of Advanced Robotic Systems, 15(1):1729881418759424, 2018.

[106] William Stallings. Computer networking with Internet protocols and technology. Pearson/Prentice Hall Upper Saddle River, NJ, USA, 2004.

[107] Matthew R Stein. Painting on the world wide web: The PumaPaint project. In Telemanipulator and Telepresence Technologies V, volume 3524, pages 201-210. International Society for Optics and Photonics, 1998.

[108] Aaron Steinfeld, Terrence Fong, David Kaber, Michael Lewis, Jean Scholtz, Alan Schultz, and Michael Goodrich. Common metrics for human-robot interaction. In Proceedings of the 1st ACM SIGCHI/SIGART conference on Human-robot interaction, pages 33-40. ACM, 2006

[109] Biljana L Risteska Stojkoska and Kire V Trivodaliev. A review of internet of things for smart home: Challenges and solutions. Journal of Cleaner Production, 140:1454-1464, 2017.

[110] Hui Suo, Jiafu Wan, Caifeng Zou, and Jianqi Liu. Security in the internet of things: a review. In Computer Science and Electronics Engineering (ICCSEE), International Conference on, volume 3, pages 648-651. IEEE, 2012.

[111] N Takanobu, Ryoji Soyama, Atsuo Takanishi, Kayoko Ohtsuki, Daisaku Ozawa, and Masatoshi Ohnishi. Remote treatment with mouth opening and closing training robot. In Intelligent Robots and Systems (IROS), IEEE/RSJ International Conference on, volume 1, pages 553-558. IEEE, 2000.

[112] Kensuke Tanaka, Eiichi Nakagawa, Masanori Ito, Naoki Mizuno, Takayoshi Yamada, Etsuro Shimizu, and Kazuyuki Kagayama. An Internet-based tele-robot environment for a time critical task. In Systems, Man, and Cybernetics (SMC), IEEE International Conference on, volume 5, pages 1106-1110, 1999.

[113] Moritz Tenorth and Michael Beetz. A unified representation for reasoning about robot actions, processes, and their effects on objects. In IEEE/RSJ International Conference on Intelligent Robots and Systems, pages 1351-1358. IEEE, 2012.

[114] Sebastian Thrun, Maren Bennewitz, Wolfram Burgard, Armin B Cremers, Frank Dellaert, Dieter Fox, Dirk Hahnel, Charles Rosenberg, Nicholas Roy, Jamieson Schulte, et al. MINERVA: A second-generation museum tour-guide robot. In Robotics and automation, IEEE International Conference on, volume 3. IEEE, 1999.

[115] Feng Tian. A supply chain traceability system for food safety based on haccp, blockchain \& internet of things. In International Conference on Service Systems and Service Management, pages 1-6. IEEE, 2017.

[116] Russell Toris, Julius Kammerl, David V Lu, Jihoon Lee, Odest Chadwicke Jenkins, Sarah Osentoski, Mitchell Wills, and Sonia Chernova. 
Robot web tools: Efficient messaging for cloud robotics. In IROS, pages 4530-4537, 2015.

[117] Cristina Turcu and Cornel Turcu. The social internet of things and the rfid-based robots. In Ultra Modern Telecommunications and Control Systems and Workshops (ICUMT), 4th International Congress on, pages 77-83. IEEE, 2012.

[118] Cristina Turcu, Cornel Turcu, and Vasile Gaitan. Integrating robots into the Internet of Things. International Journal of Circuits, Systems and Signal Processing, 6(6):430-437, 2012.

[119] Sriram Valluri, Akepati Gouri Sagar, and Siva G. Shanmugam. IOT Based Real Time Monitoring Mobile Robot. International Journal for Research in Applied Science and Engineering Technology (IJRASET), 5(V):105-109, 2017.

[120] Ovidiu Vermesan, Arne Br'oring, Elias Tragos, Martin Serrano, Davide Bacciu, Stefano Chessa, Claudio Gallicchio, Alessio Micheli, Mauro Dragone, Alessandro Saffiotti, et al. Internet of robotic things: converging sensing/actuating, hypoconnectivity, artificial intelligence and IoT Platforms. Cognitive Hyperconnected Digital Transformation: Internet of Things Intelligence Evolution, pages 97-155, 2017.

[121] Jean Vertut. Teleoperation and robotics: applications and technology, volume 3. Springer Science and Business Media, 2013.

[122] Markus Waibel, Michael Beetz, Javier Civera, Raffaello d'Andrea, Jos Elfring, Dorian Galvez-Lopez, Kai H'aussermann, Rob Janssen, JMM Montiel, Alexander Perzylo, et al. Roboearth. IEEE Robotics and Automation Magazine, 18(2):69-82, 2011.

[123] Jiafu Wan, Shenglong Tang, Zhaogang Shu, Di Li, Shiyong Wang, Muhammad Imran, and Athanasios V Vasilakos. Software-defined industrial internet of things in the context of industry 4.0. IEEE Sensors Journal, 16(20):7373-7380, 2016.

[124] Jiafu Wan, Shenglong Tang, Qingsong Hua, Di Li, Chengliang Liu, and Jaime Lloret. Context-aware cloud robotics for material handling in cognitive industrial Internet of Things. IEEE Internet of Things Journal, 5(4):2272-2281, 2018.

[125] Jennifer Wang, Erik Schluntz, Brian Otis, and Travis Deyle. A new vision for smart objects and the internet of things: Mobile robots and long-range UHF RFID sensor tags. arXiv preprint arXiv:1507.02373, 2015.

[126] Lihui Wang, Abdullah Mohammed, and Mauro Onori. Remote robotic assembly guided by $3 \mathrm{D}$ models linking to a real robot. CIRP Annals-Manufacturing Technology, 63(1):1-4, 2014.

[127] Thomas Watteyne, Xavier Vilajosana, Branko Kerkez, Fabien Chraim, Kevin Weekly, Qin Wang, Steven Glaser, and Kris Pister. Openwsn: a standards-based low-power wireless development environment. Transactions on Emerging Telecommunications Technologies, 23(5):480493, 2012.

[128] Rolf H Weber and Romana Weber. Internet of things, volume 12. Springer, 2010.

[129] Erik Wilde. Putting things to REST. 2007.

[130] Tim Winter, Pascal Thubert, Anders Brandt, Jonathan Hui, Richard Kelsey, Philip Levis, Kris Pister, Rene Struik, Jean-Philippe Vasseur, and Roger Alexander. Rpl: Ipv6 routing protocol for low-power and lossy networks. Technical report, 2012.

[131] Lamont Wood. Service robots: The next big productivity platform, 2016. https://usblogs.pwc.com/emerging-technology/service-robots-thenext-big-productivity-platform/.

[132] Yin Yuehong, Yan Zeng, Xing Chen, and Yuanjie Fan. The internet of things in healthcare: An overview. Journal of Industrial Information Integration, 1:3-13, 2016.

[133] Justyna Zander, Pieter J Mosterman, Taskin Padir, Yan Wan, and Shengli Fu. Cyber-physical systems can make emergency response smart. Procedia Engineering, 107:312-318, 2015

[134] Hubert Zangl, Anton Fuchs, Thomas Bretterklieber, Michael Moser, and Gert Holler. An investigation on wireless communication and power supply through metal tank walls. In 2008 IEEE Instrumentation and Measurement Technology Conference, pages 1452-1457. IEEE, 2008 . 